Discrete Comput Geom 33:617-644 (2005)

DOI: $10.1007 / \mathrm{s} 00454-005-1160-8$

\title{
The Envelope of Lines Meeting a Fixed Line and Tangent to Two Spheres*
}

\author{
Gábor Megyesi ${ }^{1}$ and Frank Sottile ${ }^{2}$ \\ ${ }^{1}$ Department of Mathematics, UMIST, \\ P.O. Box 88, Manchester, M60 1QD, England \\ gmegyesi@ma.umist.ac.uk \\ http://www.ma.umist.ac.uk/gm \\ ${ }^{2}$ Department of Mathematics, Texas A\&M University, \\ College Station, TX 77843, USA \\ sottile@math.tamu.edu \\ http://www.math.tamu.edu/ ssottile
}

\begin{abstract}
We study the set of lines that meet a fixed line and are tangent to two spheres and classify the configurations consisting of a single line and three spheres for which there are infinitely many lines tangent to the three spheres that also meet the given line. All such configurations are degenerate. The path to this result involves the interplay of some beautiful and intricate geometry of real surfaces in 3-space, complex projective algebraic geometry, explicit computation and graphics.
\end{abstract}

\section{Introduction}

We determine the configurations of one line and three spheres for which there are infinitely many common tangent lines to the spheres that also meet the fixed line. Configurations of four lines having infinitely many common transversal lines were described classically and Theobald [12] treated configurations of three lines and one sphere with infinitely many lines tangent to the sphere that also meet the fixed lines. The case of two lines and two spheres was solved in [7].

Besides the beautiful geometry encountered in this study, ${ }^{1}$ these questions were motivated by algorithmic problems in computational geometry. As explained in [12], problems of this type occur when one is looking for a line or ray interacting (in the sense of

\footnotetext{
* The research of the first author was supported in part by EPSRC Grant GR/S11381/01. The research of the second author was supported in part by NSF Grant DMS-0134860.

${ }^{1}$ See www. math.tamu.edu/ sottile/pages/212s/ and sottile/stories/3.Spheres.
} 
"intersecting" or in the sense of "not intersecting") with a given set of three-dimensional bodies, if the class of admissible bodies consists of polytopes and spheres. Concrete instances include visibility computations with moving viewpoints [13], controlling a laser beam in manufacturing [8] or the design of envelope data structures supporting ray shooting queries (seeking the first sphere, if any, met by a query ray) [1]. In [5], [6] and [10] the question of arrangements of four spheres in $\mathbb{R}^{3}$ with an infinite number of common tangent lines is discussed from various viewpoints. This was recently characterized [2].

We first study the common tangents to two spheres that also meet a given line and show that this one-dimensional family (a curve) of lines determines the two spheres. When the configuration of the two spheres and fixed line becomes degenerate, this curve becomes reducible and we classify its possible components. In most cases the components of the curve also determine the spheres and it is only in the remaining highly degenerate cases that there is a third sphere tangent to all the lines in a given component of the curve.

Theorem 1. Let $\ell$ be a line and let $S_{1}, S_{2}$ and $S_{3}$ be spheres in $\mathbb{R}^{3}$. Then there are infinitely many lines that meet $\ell$ and are tangent to each sphere in precisely the following cases (see Fig. 1):

(i) The spheres are tangent to each other at the same point and either (a) $\ell$ meets that point, or (b) it lies in the common tangent plane, or both. The common tangent lines are the lines in the tangent plane meeting the point of tangency.

(ii) The spheres are tangent to a cone whose apex lies on $\ell$. The common tangent lines are the ruling of the cone.

(iii) The spheres meet in a common circle and the line $\ell$ lies in the plane of that circle. The common tangents are the lines in that plane tangent to the circle.

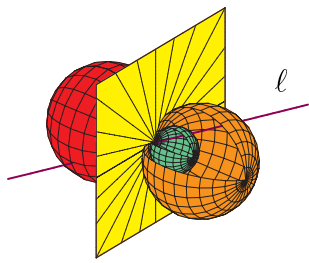

(ia)

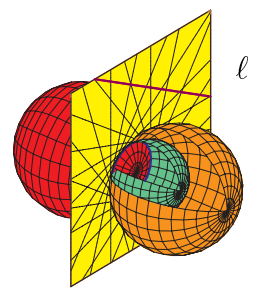

(iii)

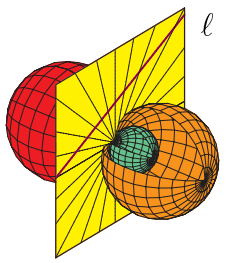

(ib)

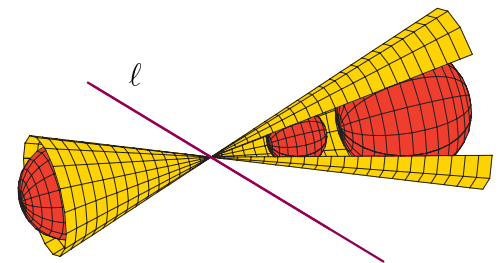

(ii)

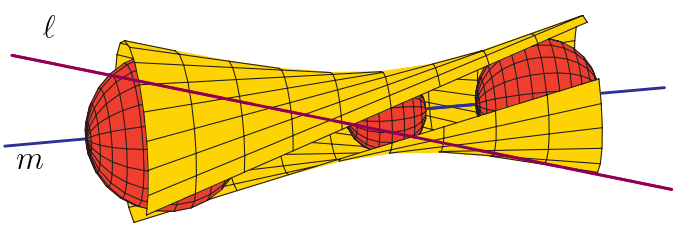

(iv)

Fig. 1. The geometry of Theorem 1 . 
(iv) The centres of the spheres lie on a line $m$ and $\ell$ is tangent to all three spheres. The common tangent lines are one ruling on the hyperboloid of revolution obtained by rotating $\ell$ about $m$.

In cases (ib), (iii) and (iv) lines parallel to $\ell$ are excluded.

The classification in real projective space is similar, except that lines parallel to $\ell$ are not excluded, and $\ell$ might be at infinity in (ib) or (iii). Also, in (ii), the cone may be a cylinder (cone with apex at infinity) with $\ell$ parallel to the cylinder. We do not know the classification over the complex numbers, as the real numbers are used in an essential way to rule out several possibilities.

The primary difficulty in proving Theorem 1 lies not in recognizing the (fairly obvious) possibilities, but rather in excluding all others. In Section 1 we give algebraic and geometric preliminaries concerning the geometry of lines tangent to spheres. In Section 2 we study the envelope of lines tangent to two spheres that meet a fixed line, describe the configurations of Theorem 1 and begin to exclude other possibilities. Specifically, we show that the algebraic subset $\tau$ of the Grassmannian consisting of common tangents to two spheres and a line is a curve of degree at most 8 . We show that it is impossible for any component $\sigma$ of $\tau$ to have degree 3, 5 or 7, and if a component $\sigma$ has degree 4,6 or 8 , then it determines the two spheres. Only in the cases of $\sigma$ having degree 1 or 2 can there be more than two spheres; these are described in Theorem 1. The intricate argument when $\sigma$ has degree 4 is treated separately in Section 3.

\section{Real Line Geometry and Plücker Coordinates}

We review some aspects of the geometry of lines in space, Plücker coordinates for lines and preliminaries from real algebraic geometry. Good general references are [3], [4] and [9].

While our main concern is lines in $\mathbb{R}^{3}$ tangent to spheres, some algebraic arguments we make apply to lines in complex projective 3 -space $\mathbb{P}^{3}$ tangent to a quadric surface, and this added generality will sometimes be necessary. Nevertheless, at key junctures the real-number nature of the answers we seek will be used in an essential way. For example, distinct concentric spheres have no common tangents in $\mathbb{R}^{3}$, but do have a two-dimensional family of common complex tangents in $\mathbb{P}^{3}$. We use $\mathbb{R}^{3}, \mathbb{R P}^{3}$ and $\mathbb{P}^{3}$ to indicate real affine space, real projective space and complex projective space respectively. In general, algebraic computation takes place in $\mathbb{P}^{3}$, and we then interpret the answers in $\mathbb{R}^{3}$ to obtain our restrictions and to describe the final configuration.

\subsection{Common Tangents in a Plane and Through a Point}

A useful warm-up is the following elementary determination of the common tangents to two spheres that lie in a plane and the common tangents through a point.

Proposition 2. Let $S_{1}$ and $S_{2}$ be spheres and let $\Pi$ be a plane $\mathbb{R}^{3}$ such that $\Pi \cap S_{1} \neq$ $\Pi \cap S_{2}$, as subsets of $\mathbb{R}^{3}$. Then there are four (complex) common tangents to $S_{1}$ and $S_{2}$ that lie in $\Pi$, counted with multiplicity. Furthermore, if $\Pi$ is not tangent to either sphere, then these common tangents determine the circles $\Pi \cap S_{1}$ and $\Pi \cap S_{2}$. 

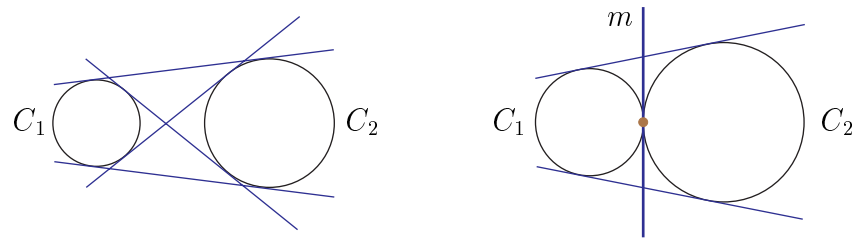

Fig. 2. Lines tangent to two circles.

Proof. If $\Pi$ is not tangent to $S_{1}$, then $\Pi \cap S_{1}$ is a smooth conic whose tangents form a conic in the dual projective plane $\widehat{\Pi}$. If $\Pi$ is tangent to $S_{1}$ at a point $q$ then the "tangents" (lines with a point of double contact) to $\Pi \cap S_{1}$ are the set of lines in $\Pi$ through $q$, counted with multiplicity 2 , a degenerate conic in $\widehat{\Pi}$. Thus the common (complex) tangents to $\Pi \cap S_{1}$ and $\Pi \cap S_{2}$ are the intersection of two conics in $\widehat{\Pi}$, and so there are four, counted with multiplicity.

Suppose that $\Pi$ is not tangent to either sphere. Then the four common tangents determine the circles $C_{1}:=\Pi \cap S_{1}$ and $C_{2}:=\Pi \cap S_{2}$ : three distinct (complex) lines in $\Pi$ are common tangents to four circles. The fourth common tangent selects two of these four circles. This is clear when there are four distinct common tangents (see Fig. 2).

Otherwise, $C_{1}$ and $C_{2}$ are tangent at the same point of the common tangent having multiplicity 2 , and this additional information determines $C_{1}$ and $C_{2}$.

Proposition 3. Let $S_{1}$ and $S_{2}$ be spheres and let $p$ be a point in $\mathbb{R}^{3}$ such that the cone with apex $p$ tangent to $S_{1}$ is distinct from the cone with apex $p$ tangent to $S_{2}$. Then there are four (possibly complex) common tangents to $S_{1}$ and $S_{2}$ that meet $p$, counted with multiplicity.

Proof. Let $C_{1}$ and $C_{2}$ be the cones with apex $p$ tangent to $S_{1}$ and $S_{2}$, respectively. If $\Pi$ is a plane not containing $p$, then $\Pi \cap C_{1}$ and $\Pi \cap C_{2}$ are plane conics, distinct by hypothesis. These conics meet in four points, counted with multiplicity, and these points of intersection are where the common tangents meet $\Pi$.

We display a configuration of two cones with four real common tangent lines below:

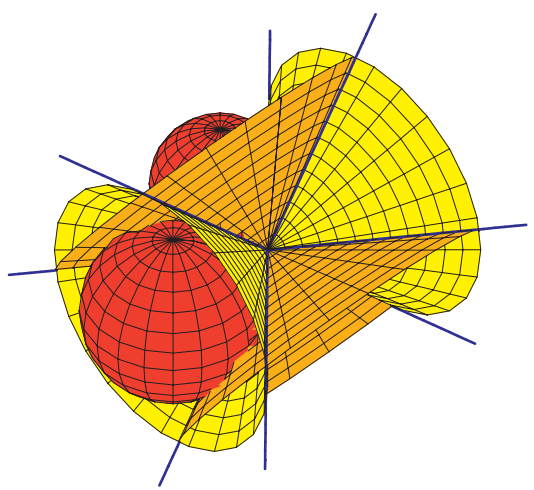




\subsection{Plücker Coordinates for Lines}

The Grassmannian $\mathbb{G}$ (or Klein quadric [9, Section 2.1]) is the set of lines in complex projective 3-space $\mathbb{P}^{3}$. The line $\ell$ between two points $x=\left(x_{0}, x_{1}, x_{2}, x_{3}\right)^{T}$ and $y=$ $\left(y_{0}, y_{1}, y_{2}, y_{3}\right)^{T}$ in $\mathbb{P}^{3}$ is represented (non-uniquely) by the $4 \times 2$ matrix whose columns are the vectors $x$ and $y$. A unique representation is given by its Plücker vector $p_{\ell}:=$ $\left(p_{01}, p_{02}, p_{03}, p_{12}, p_{13}, p_{23}\right)^{T} \in \mathbb{P}^{5}$, where $p_{i j}:=x_{i} y_{j}-x_{j} y_{i}$. The coordinates of the Plücker vector satisfy the Plücker relation

$$
0=p_{03} p_{12}-p_{02} p_{13}+p_{01} p_{23},
$$

and $\mathbb{G}$ is the set of points in $\mathbb{P}^{5}$ which satisfy this relation. Moreover, a line $\ell$ is in $\mathbb{R} \mathbb{P}^{3}$ if and only if its Plücker vector lies in $\mathbb{R} \mathbb{P}^{5} \cap \mathbb{G}$, the real part of the Grassmannian.

A line $\ell$ meets another line $\ell^{\prime}$ if and only if their Plücker vectors $p$ and $p^{\prime}$ satisfy

$$
p_{01} p_{23}^{\prime}-p_{02} p_{13}^{\prime}+p_{03} p_{12}^{\prime}+p_{12} p_{03}^{\prime}-p_{13} p_{02}^{\prime}+p_{23} p_{01}^{\prime}=0 .
$$

Geometrically, this means that the set of lines meeting a given line $\ell$ is the intersection of $\mathbb{G}$ with a hyperplane $\Lambda_{\ell}$ in $\mathbb{P}^{5}$. For example, the $x$-axis (defined by the vanishing of the last two coordinates) is the span of the points $(1,0,0,0)^{T}$ and $(0,1,0,0)^{T}$, and thus has Plücker vector $(1,0,0,0,0,0)^{T}$ as $p_{01}=1$ and the other coordinates vanish. The set of lines that meet the $x$-axis is then given by $p_{23}=0$.

Each hyperplane $\Lambda_{\ell}$ is tangent to $\mathbb{G}$ at the point $p_{\ell} \in \mathbb{G}$. The left-hand side of (1.2) defines a symmetric bilinear form $\left\langle p, p^{\prime}\right\rangle$ on $\mathbb{P}^{5}$, identifying it with the dual projective space of hyperplanes in $\mathbb{P}^{5}$, and $\mathbb{G}$ with its dual $\mathbb{G}^{*}$.

Remark 4. Let $H \subset \mathbb{P}^{5}$ be a three-dimensional linear subspace. The set of hyperplanes containing $H$ is a line $h$ in the dual projective space. If the intersection $h \cap \mathbb{G}^{*}$ consists of two points, then these correspond to two hyperplanes $\Lambda_{\ell}$ and $\Lambda_{\ell^{\prime}}$. Thus $H=\Lambda_{\ell} \cap \Lambda_{\ell^{\prime}}$ and $H \cap \mathbb{G}$ parametrizes lines meeting $\ell$ and $\ell^{\prime}$. If $H$ is real, then either both $\ell$ and $\ell^{\prime}$ are real or else they are complex conjugate to each other. If however $h \cap \mathbb{G}^{*}$ is a single point, so that $h$ is tangent to $\mathbb{G}^{*}$, then $H$ is tangent to $\mathbb{G}$ and $H \cap \mathbb{G}$ is a singular quadric-a cone over a plane conic with apex $p_{\ell}$, the point of tangency.

Plücker coordinates afford a compact characterization of the lines tangent to a given sphere and more generally tangent to a quadric surface. We follow the presentation of [7] and [11]. Identify a quadric $x^{T} Q x=0$ in $\mathbb{P}^{3}$ with the symmetric $4 \times 4$ matrix $Q$. Thus the sphere with centre $\left(x_{0}, y_{0}, z_{0}\right)^{T} \in \mathbb{R}^{3}$ and radius $r$ described in $\mathbb{P}^{3}$ by $\left(x-x_{0} w\right)^{2}+\left(y-y_{0} w\right)^{2}+\left(z-z_{0} w\right)^{2}=r^{2} w^{2}$ is identified with the matrix

$$
\left(\begin{array}{cccc}
x_{0}^{2}+y_{0}^{2}+z_{0}^{2}-r^{2} & -x_{0} & -y_{0} & -z_{0} \\
-x_{0} & 1 & 0 & 0 \\
-y_{0} & 0 & 1 & 0 \\
-z_{0} & 0 & 0 & 1
\end{array}\right) .
$$

The second exterior power of a $4 \times 4$ matrix $Q$ is the $6 \times 6$ matrix $\wedge^{2} Q$ whose entries are the $2 \times 2$ minors of $Q$. Its rows and columns have the same index set as do Plücker vectors. 
Proposition 5 (Proposition 5.2 of [10]). A line $\ell \subset \mathbb{P}^{3}$ is tangent to a quadric $Q$ if and only if its Plücker vector $p_{\ell}$ lies on the quadric hypersurface in $\mathbb{P}^{5}$ defined by $\wedge^{2} Q$,

$$
p_{\ell}^{T}\left(\wedge^{2} Q\right) p_{\ell}=0 .
$$

For a sphere with radius $r$ and centre $\left(x_{0}, y_{0}, z_{0}\right)^{T} \in \mathbb{R}^{3}$ the quadratic form $p_{\ell}^{T}\left(\wedge^{2} Q\right)$ $p_{\ell}$ is

$$
\left(\begin{array}{l}
p_{01} \\
p_{02} \\
p_{03} \\
p_{12} \\
p_{13} \\
p_{23}
\end{array}\right)^{T}\left(\begin{array}{cccccc}
y_{0}^{2}+z_{0}^{2}-r^{2} & -x_{0} y_{0} & -x_{0} z_{0} & y_{0} & z_{0} & 0 \\
-x_{0} y_{0} & x_{0}^{2}+z_{0}^{2}-r^{2} & -y_{0} z_{0} & -x_{0} & 0 & z_{0} \\
-x_{0} z_{0} & -y_{0} z_{0} & x_{0}^{2}+y_{0}^{2}-r^{2} & 0 & -x_{0} & -y_{0} \\
y_{0} & -x_{0} & 0 & 1 & 0 & 0 \\
z_{0} & 0 & -x_{0} & 0 & 1 & 0 \\
0 & z_{0} & -y_{0} & 0 & 0 & 1
\end{array}\right)\left(\begin{array}{l}
p_{01} \\
p_{02} \\
p_{03} \\
p_{12} \\
p_{13} \\
p_{23}
\end{array}\right) .
$$

We use the relation between the intrinsic geometry of $\mathbb{G}$ and families of lines in $\mathbb{P}^{3}$. This begins with the following identification of linear subspaces lying in $\mathbb{G}$.

Proposition 6 [9, pp. 141-143]. The set of lines in $\mathbb{P}^{3}$ meeting a given point $p$ is a two-dimensional linear subspace contained in $\mathbb{G}$. Dually, the set of lines lying in a given 2-plane $\Pi$ is a two-dimensional linear subspace contained in $\mathbb{G}$, and any 2-plane contained in $\mathbb{G}$ is one of these two types.

A one-dimensional linear subspace contained in $\mathbb{G}$ consists exactly of the set of lines in $\mathbb{P}^{3}$ which contain a fixed point $p$ and lie in a fixed plane $\Pi$.

\subsection{The Envelope of a Curve in $\mathbb{G}$}

The union $\Sigma$ of the lines in $\mathbb{P}^{3}$ determined by a curve $\sigma$ in $\mathbb{G}$ is a ruled surface, which we call the envelope of $\sigma$. The lines in $\sigma$ are a ruling of $\Sigma$. For example, there is an irreducible octic (degree 8) curve $\sigma$ in $\mathbb{G}$ consisting of lines meeting the $x$-axis that are also tangent to two spheres centred at $(0, \pm 2,0)$ with respective radii 1 and $\sqrt{3}$. Its envelope $\Sigma$ is displayed in Fig. 3, where we also show the curves of self-intersection of $\Sigma$. The real points of the envelope $\Sigma$ of Fig. 3 appear to have two irreducible components, and thus contain two distinct families of lines. This is a real-number illusion. The complex curve is irreducible (in fact smooth and connected), but its real points consist of two topological components which cannot be separated by an algebraic function.

Suppose that $\sigma$ is an irreducible curve in $\mathbb{G}$. Unless its envelope $\Sigma$ is a plane $\Pi$, the degree of the surface $\Sigma \subset \mathbb{P}^{3}$ equals the degree of the curve $\sigma$ [9, Theorem 5.2.8], and this common degree is the intersection multiplicity of $\Sigma$ with a line $m$ not contained in $\Sigma$. If a plane $\Pi$ is not contained in $\Sigma$, then the degree of $\Sigma$ equals the degree of the plane curve $\Sigma \cap \Pi$, if we keep track of the multiplicities of its components. These methods will also determine the degree of a curve $\sigma$, even when $\sigma$ is reducible or has components whose envelope is a plane, if we are careful with the multiplicities.

We make free use of continuity arguments from algebraic geometry. Specifically, the intersection multiplicity of a generic configuration is a lower bound for the multiplicity of a special configuration. 


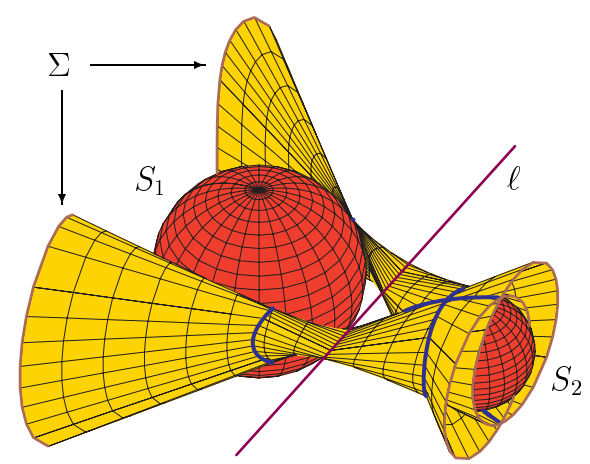

Fig. 3. An envelope.

Example 7. An important case of ruled surfaces is when $\sigma$ is an irreducible plane conic. If the plane of $\sigma$ lies in $\mathbb{G}$, then there are two possibilities, by Proposition 6. When the plane consists of lines through a point $p \in \mathbb{P}^{3}$, then $\Sigma$ will be a cone (over a conic) with apex $p$, or a cylinder if $p$ lies at infinity. When the plane consists of lines in a plane $\Pi \subset \mathbb{P}^{3}$, then $\sigma$ will be the set of lines tangent to a conic in $\Pi$.

The most interesting case is when the plane of $\sigma$ does not lie in $\mathbb{G}$. A smooth quadric surface in $\mathbb{P}^{3}$ is ruled by two families of lines and each line in one family meets each line in the other, but no other lines in its own family. Any smooth conic $\sigma \subset \mathbb{G}$ whose plane does not lie in $\mathbb{G}$ corresponds to a ruling of some quadric surface $Q$ [9, Proposition 3.3.1]. A plane tangent to a point of $Q$ meets $Q$ in two lines, one from each ruling.

Suppose that $Q \subset \mathbb{R}^{3}$ is a real quadric surface. If $Q$ contains even a single real line, then $Q$ is either a hyperbolic paraboloid or a hyperboloid of one sheet, and the two rulings are real. Otherwise, the rulings are complex conjugate to each other. Any curve $\gamma$ on $Q$ has a bidegree $(a, b)$, where $a$ is the intersection multiplicity of $\gamma$ with a general line in one family and $b$ is the intersection multiplicity with a general line in the other. If $\gamma$ is the intersection of $Q$ with a hypersurface of degree $d$, then its bidegree is $(d, d)$. Real curves $\gamma$ on a sphere $Q$ will have symmetric bidegree, as complex conjugation preserves $\gamma$ but interchanges the rulings of $Q$.

\subsection{Lines Tangent to a Sphere that Meet a Fixed Line}

We study the correspondence between the points of a sphere and the lines tangent to it that meet a fixed line. Our goal is Lemma 8, which relates the degree of a curve $\gamma$ on the sphere to the curve $\sigma$ parametrizing lines which are tangent to the sphere at points of $\gamma$ and also meet the fixed line.

Let $S$ be a sphere and let $\ell$ be a line in $\mathbb{R}^{3}$. The tangent plane at a general point $p$ of $S$ meets $\ell$ in a single point, and thus there is a unique tangent line to $S$ at $p$ meeting $\ell$. This defines an algebraic correspondence between points $p$ of $S$ and lines tangent to $S$ that meet $\ell$. This correspondence is given by polynomials that are quadratic in the coordinates $\left(x_{0}, y_{0}, z_{0}\right)^{T}$ of the point $p$. 
To see this, suppose that $S$ has centre $(a, b, c)^{T} \in \mathbb{R}^{3}$ and radius $r$. Then the tangent plane to $S$ at a point $p=\left(x_{0}, y_{0}, z_{0}\right)^{T}$ is defined by the linear equation

$$
\left(x_{0}-a, y_{0}-b, z_{0}-c\right) \cdot(x, y, z)^{T}=r^{2}+a\left(x_{0}-a\right)+b\left(y_{0}-b\right)+c\left(z_{0}-c\right) .
$$

Write this compactly in vector form as $M \cdot(x, y, z)^{T}=\mu$, where $M=\left(x_{0}-a, y_{0}-b, z_{0}-c\right)$ and $\mu=r^{2}+M \cdot(a, b, c)^{T}$.

Suppose that the line $\ell$ is defined by the intersection of two linear equations:

$$
\Lambda_{1} \cdot(x, y, z)^{T}=\lambda_{1} \quad \text { and } \quad \Lambda_{2} \cdot(x, y, z)^{T}=\lambda_{2} .
$$

Then the tangent plane to $S$ at $p$ meets $\ell$ at the solution of the linear system

$$
\left(\begin{array}{c}
M \\
\Lambda_{1} \\
\Lambda_{2}
\end{array}\right) \cdot\left(\begin{array}{c}
x \\
y \\
z
\end{array}\right)=\left(\begin{array}{c}
\mu \\
\lambda_{1} \\
\lambda_{2}
\end{array}\right)
$$

This solution is

$$
\left(\begin{array}{l}
x_{1} \\
y_{1} \\
z_{1}
\end{array}\right)=\left(\begin{array}{c}
M \\
\Lambda_{1} \\
\Lambda_{2}
\end{array}\right)^{-1} \cdot\left(\begin{array}{c}
\mu \\
\lambda_{1} \\
\lambda_{2}
\end{array}\right)=\operatorname{Ad}\left(\begin{array}{c}
M \\
\Lambda_{1} \\
\Lambda_{2}
\end{array}\right) \cdot\left(\begin{array}{c}
\mu \\
\lambda_{1} \\
\lambda_{2}
\end{array}\right) / \operatorname{det}\left(\begin{array}{c}
M \\
\Lambda_{1} \\
\Lambda_{2}
\end{array}\right) .
$$

The Plücker coordinates of the line in $\mathbb{P}^{3}$ tangent to $S$ at $\left(x_{0}, y_{0}, z_{0}\right)^{T}$ that meets $\ell$ are the $2 \times 2$ minors of

$$
\left[\begin{array}{cccc}
1 & x_{0} & y_{0} & z_{0} \\
1 & x_{1} & y_{1} & z_{1}
\end{array}\right]^{T}
$$

By (1.5), the coordinates $x_{1}, y_{1}, z_{1}$ are rational functions of $x_{0}, y_{0}, z_{0}$ whose numerators are linear and which have the same (linear in $x_{0}, y_{0}, z_{0}$ ) denominator, the determinant in (1.5). Clearing denominators shows that the Plücker coordinates of the line tangent to $S$ at $\left(x_{0}, y_{0}, z_{0}\right)^{T}$ that meet $\ell$ are quadratic polynomials in $x_{0}, y_{0}, z_{0}$.

Let $\Phi$ be this algebraic correspondence between (complex) points of $S$ and tangent lines:

$$
\Phi:\{\text { points of } S\}--\rightarrow \text { \{lines tangent to } S \text { meeting } \ell \text { \}. }
$$

The broken arrow is used in algebraic geometry to indicate that $\Phi$ does not define a function at all points of $S$. Such a correspondence that is $1-1$ almost everywhere is called a birational equivalence. We describe the loci where $\Phi$ is not 1-1 in geometric terms. For this, we work in complex projective space. This description is valid more generally for quadrics in $\mathbb{P}^{3}$.

A special point of $S$ is a point either lying on $\ell$ or whose tangent plane contains $\ell$. Every line tangent to $S$ at a special point meets $\ell$, and these are exactly the points where $\Phi$ is not well defined. In particular, the correspondence $\Phi$ associates a special point to the one-dimensional subspace of $\mathbb{G}$ consisting of those lines tangent to $S$ at the special point. Conversely, if $m$ is a line lying in $S$ that meets $\ell$ (a special line), then $\Phi$ maps every point of $m$ to $m$. 
If $\ell$ is tangent to $S$ at a point $p$, then $p$ is the only special point and the two lines lying in $S$ in the tangent plane at $p$ are the only special lines. Otherwise $\ell$ meets $S$ in two points and there are two planes through $\ell$ tangent to $S$, so there are four special points $p_{1}, p_{2}, p_{3}, p_{4}$. The four lines in $S$ that meet $\ell$ are the special lines. Two special points are always real and the other two are a complex conjugate pair, the special lines always form two complex conjugate pairs. In the terminology of algebraic geometry, we say that $\Phi$ blows up the special points and blows down the special lines.

Let $\gamma \subset S$ be an irreducible curve, not a special line. The proper transform $\Phi(\gamma)$ of $\gamma$ is the closure of the image of $\gamma_{0}$ under $\Phi$, where $\gamma_{0}$ is $\gamma$ with any special points removed. In Lemma 19, we will need to know the degree of $\Phi(\gamma)$, where $\gamma$ is rational. This determination uses more machinery from algebraic geometry than is needed by other parts of this paper.

Lemma 8. Let $\gamma \subset S$ be a rational curve, not one of the special lines. Then the degree of the proper transform $\Phi(\gamma)$ of $\gamma$ is

(i) $2 \operatorname{deg} \gamma-\sum_{k=1}^{4}$ mult $_{p_{k}} \gamma$ if there are four special points $p_{1}, p_{2}, p_{3}, p_{4}$.

(ii) $2 \operatorname{deg} \gamma-2$ mult $_{p} \gamma$ if there is a unique special point $p$.

Proof. As $\gamma$ is rational, there is a rational map $v: \mathbb{P}^{1} \rightarrow \gamma \subset S \subset \mathbb{P}^{3}$, which is an isomorphism outside a finite set. Let us choose homogeneous coordinates on $\mathbb{P}^{1}$. Any rational map $\varphi: \mathbb{P}^{1} \rightarrow \mathbb{P}^{n}$ is defined by an $(n+1)$-tuple of homogeneous polynomials of the same degree. The polynomials themselves are not uniquely defined, but their ratios are, and we can multiply or divide them by a common factor. If we divide all of them by their greatest common divisor to obtain an $(n+1)$-tuple of coprime polynomials, then they do not all vanish at the same point of $\mathbb{P}^{1}$, so $\varphi$ is defined everywhere, it is a morphism. Furthermore, if $\varphi$ is generically $1-1$, i.e., it is $1-1$ outside a finite set, then the degree of the coprime polynomials defining $\varphi$ is the degree of the image $\varphi\left(\mathbb{P}^{1}\right)$ in $\mathbb{P}^{n}$.

This means that $v$ can be defined by coprime homogeneous polynomials of degree $\operatorname{deg} \gamma$ on $\mathbb{P}^{1}$. Let $\Phi_{i j}, 0 \leq i<j \leq 3$, be the quadratic polynomials defining the map $\Phi$ (1.6). Under $v$, they pull back to homogeneous polynomials $\Psi_{i j}, 0 \leq i<j \leq 3$, of degree $2 \operatorname{deg} \gamma$ on $\mathbb{P}^{1}$, which define the map $\Psi=\Phi \circ v$ whose image agrees with $\Phi(\gamma)$.

The special points are exactly the points of $S$ where all the forms $\Phi_{i j}(0 \leq i<j \leq 3)$ vanish. If $\gamma$ does not pass through any of the special points, then the forms $\Psi_{i j}(0 \leq$ $i<j \leq 3$ ) do not all vanish at the same point of $\mathbb{P}^{1}$, so they are coprime, and therefore the degree of $\Psi\left(\mathbb{P}^{1}\right)=\Phi(\gamma)$ is $2 \operatorname{deg} \gamma$. If $\gamma$ does pass through a special point, then all the $\Psi_{i j}(0 \leq i<j \leq 3)$ vanish at the inverse image of that point on $\mathbb{P}^{1}$, so they have a common factor which can be cancelled. The degree of the image drops by the degree of the common factor, and it is this degree that we need to calculate.

The equation $\Phi_{i j}=0$ defines a curve on $S$ unless $\Phi_{i j}$ is identically zero. Assume that this curve meets $\gamma$ at a point $q$. Let $s \in \mathbb{P}^{1}$ be a point such that $v(s)=q$, and let $\gamma^{\prime}$ be the branch of $\gamma$ at $q$ corresponding to $s$. The intersection multiplicity of $\Phi_{i j}=0$ with $\gamma^{\prime}$ is the same as the order of vanishing of $\Psi_{i j}$ at $s$.

In case (i), each polynomial $\Phi_{i j}(0 \leq i<j \leq 3)$ vanishes at the four special points. The multiplicity of a branch of $\gamma$ at a special point $p_{k}$ is a lower bound for the intersection multiplicity of the curve $\gamma$ at $p_{k}$, so each polynomial $\Psi_{i j}(0 \leq i<j \leq 3)$ vanishes 
at least to that order at the corresponding point of $v^{-1}\left(p_{k}\right)$. Therefore the polynomials $\Psi_{i j}(0 \leq i<j \leq 3)$ have a common factor of degree at least $\sum_{k=1}^{4}$ mult $_{p_{k}} \gamma$. If we choose coordinates such that $\ell$ is the $x$-axis and the centre of $S$ lies on the $y$-axis, then one may check by direct calculation that the curves defined by $\Phi_{02}=0$ and $\Phi_{13}=0$ are smooth at all of the special points and have different tangent directions at each special point. If $s$ is a point of $\mathbb{P}^{1}$ such that $v(s)$ is a special point $p_{k}$ and $\gamma^{\prime}$ is the corresponding branch of $\gamma$ at $p_{k}$, then the intersection multiplicity of $\gamma^{\prime}$ at $p_{k}$ with at least one of the curves $\Phi_{02}=0$ and $\Phi_{13}=0$ is exactly mult $p_{k} \gamma^{\prime}$, so the greatest common divisor of the $\Psi_{i j}(0 \leq i<j \leq 3)$ vanishes at $s$ to order mult $p_{k} \gamma^{\prime}$ exactly. Therefore the degree of greatest common divisor of the $\Psi_{i j}(0 \leq i<j \leq 3)$ is $\sum_{k=1}^{4}$ mult $_{p_{k}} \gamma$, which proves the lemma for case (i).

In case (ii) we can choose coordinates such that $\ell$ is the $x$-axis and $S$ has affine equation $x^{2}+(y-1)^{2}+z^{2}=1$. Then $\Phi_{01}=\left(x^{2}-y^{2}-z^{2}\right) / 2$ and $\Phi_{03}=x z$. They both define curves with a simple node at the special point $p=(0,0,0)^{T}$, and their tangent directions there are different. If $s \in v^{-1}(p)$ and $\gamma^{\prime}$ is the corresponding branch of $\gamma$ at $p$, then the intersection multiplicity of $\gamma^{\prime}$ at $p$ with at least one of the curves $\Phi_{01}=0$ and $\Phi_{03}=0$ is exactly 2 mult $_{p} \gamma^{\prime}$, so at least one of $\Psi_{01}$ and $\Psi_{03}$ vanishes to order exactly 2 mult $_{p} \gamma^{\prime}$ at $s$. Therefore the degree of the greatest common divisor of the $\Psi_{i j}$ $(0 \leq i<j \leq 3)$ is 2 mult $_{p} \gamma$, which proves the lemma for case (ii).

The conclusion of Lemma 8 also holds for a general curve $\gamma$, but we only need the result for rational curves. The proof for general curves is more sophisticated as we do not have a rational parametrization. We may either work with a local parameter at points of the normalization of $\gamma$, or consider the multiplicity of basepoints in the rational map $\Phi$.

\section{The Envelope of Lines Meeting a Fixed Line and Tangent to Two Spheres}

Let $\ell$ be a line and let $S_{1}$ and $S_{2}$ be spheres in $\mathbb{R}^{3}$. We assume that the spheres have infinitely many common real tangents, so that in particular neither sphere contains the other. We begin to describe the envelope of lines meeting $\ell$ that are also tangent to two spheres, $S_{1}$ and $S_{2}$. We also outline and begin our proof of Theorem 1 .

Let $\tau$ be the algebraic subset of the Grassmannian $\mathbb{G}$ defined by the linear equation $\Lambda_{\ell}$ and two quadratic equations of the form (1.4) for lines to be tangent to $S_{1}$ and $S_{2}$. Then $\tau$ parametrizes the common tangents to the spheres $S_{1}$ and $S_{2}$ that also meet the fixed line $\ell$, retaining information about algebraic multiplicities.

Theorem 9. $\tau$ is a curve of degree 8 that determines the spheres $S_{1}$ and $S_{2}$.

Proof. Let $m$ be a line in $\mathbb{R}^{3}$ that meets $\ell$ in a point $p$. Then the common tangents to $S_{1}$ and $S_{2}$ that meet both $m$ and $\ell$ are either the common tangents to $S_{1}$ and $S_{2}$ that lie in the plane $\Pi$ spanned by $\ell$ and $m$, or the common tangents to $S_{1}$ and $S_{2}$ passing through the point $p$. When the line $m$ is chosen so the point $p$ and the plane $\Pi$ satisfy the hypotheses of Propositions 2 and 3, then there will be four of each type, counted with 
multiplicity. Thus the hyperplane $\Lambda_{m}$ in Plücker space meets $\tau$ in eight points, counted with multiplicity. This implies that $\tau$ is a curve of degree 8 .

By Proposition 2, given a plane $\Pi$ through $\ell$ that is not tangent to either sphere, the tangents to $S_{1}$ and $S_{2}$ lying in $\Pi$ determine the circles $\Pi \cap S_{1}$ and $\Pi \cap S_{2}$. Letting the plane $\Pi$ vary shows that $\tau$ determines the spheres $S_{1}$ and $S_{2}$.

Corollary 10. There cannot be infinitely many tangent lines to three spheres that also meet a given line, if the line and any two of the spheres are in general position so that the set of common tangents $\tau$ to the spheres that meet $\ell$ is an irreducible curve.

By Corollary 10, if we have a third sphere $S_{3}$ tangent to infinitely many of the lines in $\tau$, that set of lines is a proper subset of $\tau$. Since this infinite set is an algebraic subvariety of the curve $\tau$, we conclude that $\tau$ is reducible and the subset of $\tau$ consisting of lines tangent to $S_{3}$ is a union of some, but not all, components of $\tau$, together with possibly finitely many other points of $\tau$. We study the envelope $T$ of the curve $\tau$, a surface of degree 8 .

Theorem 11. $T$ is singular along the line $\ell$. Furthermore, if $\Pi$ is a plane through $\ell$, but not lying in $T$, then $\ell$ is a component of multiplicity at least 4 in the degree 8 curve $\Pi \cap T$. If $\ell$ is not tangent to both spheres, then this multiplicity is exactly 4.

Proof. By Proposition 3, there will be four common complex tangents to the two spheres that meet a fixed point in $\mathbb{P}^{3}$. Thus when the line and two spheres are in general position, there are four lines in $T$ that meet at a general point $p$ of $\ell$. In particular, $\ell$ lies on $T$ and four branches of $T$ meet along $\ell$, and so $T$ is singular along $\ell$. Since four branches of $T$ meet along $\ell$, if $\Pi$ is a plane through $\ell$ that is not contained in $T$, then $\ell$ is a component of multiplicity at least 4 in the degree 8 curve $T \cap \Pi$. The other components of $T \cap \Pi$ are common tangents to the circles $S_{1} \cap \Pi$ and $S_{2} \cap \Pi$. Since there are four such common tangents counted with multiplicity, these common tangents contribute a multiplicity of at least 4 to $T \cap \Pi$.

Thus, if $\ell$ is not tangent to both spheres, then both $\ell$ and the set of common tangents to the circles $S_{1} \cap \Pi$ and $S_{2} \cap \Pi$ each contributes a multiplicity of 4 to $T \cap \Pi$.

We classify the possible components $\sigma$ of $\tau$ that can occur.

Theorem 12. Suppose that $\ell, S_{1}$ and $S_{2}$ are, respectively, a line and two spheres in $\mathbb{R}^{3}$, and that $\sigma$ is a reduced, irreducible curve in the Grassmannian $\mathbb{G}$ such that every line in $\sigma$ meets $\ell$ and is tangent to both of $S_{1}$ and $S_{2}$. Then $\sigma$ has degree either 1, 2, 4, 6 or 8 . Furthermore, if $\sigma$ has degree 1 , then it is a component of multiplicity 2 or 4 in the curve $\tau$ of all lines in the Grassmannian that meet $\ell$ and are tangent to both $S_{1}$ and $S_{2}$.

Our strategy to prove Theorem 1 is now clear: We shall examine the geometry of the configurations of $\ell, S_{1}$ and $S_{2}$ when the component $\sigma$ has degree 1,2, 4 or 6 , and in each case describe completely the configurations of a third sphere tangent to all the lines in $\sigma$. The cases when $\sigma$ has degree 1 or 2 are detailed in Theorem 1 , and when the degree 
is 4 or 6 , we show there are no possibilities for the third sphere. Theorem 9 covers the case when the component has degree 8 .

We prove Theorem $12 \mathrm{in}$ this section. The main point will be to exclude the possibilities of $\sigma$ having degree 3,5 or 7. Along the way, we also begin the proof of Theorem 1 by studying in detail the geometry of the envelope of lines that meet $\ell$ and are tangent to both $S_{1}$ and $S_{2}$. As before, we first work in complex projective space to describe the possible components algebraically, and then consider the situation in $\mathbb{R}^{3}$ to exclude further components and to describe the configuration in $\mathbb{R}^{3}$.

\subsection{The Degree of $\sigma$ Is 1}

We have the following trichotomy.

Theorem 13. Let $\sigma$ be a one-dimensional linear subspace in $\mathbb{G}$ consisting of the lines lying in a plane $\Pi$ that meet a point $p \in \Pi$ such that every line in $\sigma$ meets a fixed line $\ell$ and is tangent to two spheres $S_{1}$ and $S_{2}$. Then $S_{1}$ and $S_{2}$ are tangent to each other at the point $p$ with common tangent plane $\Pi$, and we further have one of

(i) $p \in \ell \not \subset \Pi$,

(ii) $p \notin \ell \subset \Pi$, or

(iii) $p \in \ell \subset \Pi$.

In (iii), $\sigma$ is a component of multiplicity 4 in the degree 8 curve $\tau$ of common tangents to $S_{1}$ and $S_{2}$ that meet $\ell$, and in the remaining cases $\sigma$ is a component of multiplicity 2 .

Theorem 13 proves statement (i) of Theorem 1: Suppose that $\sigma$ is a one-dimensional linear subspace in $\mathbb{G}$, every line of which meets $\ell$ and is tangent to three spheres $S_{1}$, $S_{2}$ and $S_{3}$. By Theorem 13, the three spheres are mutually tangent at the point $p$ with common tangent plane $\Pi$, where $\sigma$ consists of the lines in $\Pi$ that meet $p$, and we further have that $\ell$ either meets $p$ or $\ell$ lies in $\Pi$, or both.

Proof. Our proof of this statement is entirely algebraic, working in complex projective space and in the Grassmannian $\mathbb{G}$. Suppose that $\sigma$ is a one-dimensional linear subspace lying in $\mathbb{G}$. By Proposition $6, \sigma$ consists of the lines in a plane $\Pi$ that contain a given point $p \in \Pi$. If each line in $\sigma$ is tangent to a sphere $S$, then each line is tangent to the conic $S \cap \Pi$. The only possibility is that $S$ is tangent to $\Pi$ at the point $p$. Similarly, if every line in $\sigma$ meets a fixed line $\ell$, then either (i) $\ell$ contains the point $p$ or else (ii) it lies in the plane $\Pi$ or (iii) both.

We determine the multiplicity of $\sigma$ in each of these three cases. Suppose that $p$ is the origin in $\mathbb{R}^{3}$ and $\Pi$ is the $y z$-plane, so that $\sigma$ is defined by the equations $p_{01}=p_{12}=$ $p_{13}=p_{23}=0$. Then $S_{1}$ and $S_{2}$ have their centres along the $x$-axis, say at points $x_{1}$ and $x_{2}$ (with radii $\left|x_{1}\right|$ and $\left|x_{2}\right|$ ). The quadratic forms (1.4) defined by $\wedge^{2} S_{i}$ become

$$
-x_{i}^{2} p_{01}^{2}-2 x_{i}\left(p_{02} p_{12}+p_{03} p_{13}\right)+p_{12}^{2}+p_{13}^{2}+p_{23}^{2}, \quad \text { for } \quad i=1,2 \text {. }
$$

We see that the quadric hypersurfaces defined by $\wedge^{2} S_{1}$ and $\wedge^{2} S_{2}$ have the same tangent plane at every point $\left(0, p_{02}, p_{03}, 0,0,0\right)^{T}$ of $\sigma$ and therefore $\sigma$ has multiplicity at least 
2 in $\tau$. We show that this multiplicity is exactly 2 in cases (i) and (ii) by considering degenerate configurations.

In case (i) $(p \in \ell)$ suppose that $\ell$ is the $x$-axis and let $\tau$ be the curve of common tangents to $S_{1}$ and $S_{2}$ that also meet $\ell$. We compute the intersection of $\tau$ with the hyperplane $\Lambda_{m}$ in Plücker space corresponding to a line $m$ meeting $\ell$ in a point $q$ distinct from the origin $p$ and from the apex of the cone tangent to both $S_{1}$ and $S_{2}$. A line meets both $\ell$ and $m$ if it lies in the plane $\Pi$ they span or if it meets $q$. As in the proof of Theorem 9, there will be four common tangents to $S_{1}$ and $S_{2}$ meeting $q$ (but none lies in $\sigma$ ) and four common tangents lying in $\Pi$. Since $\Pi \cap S_{1}$ and $\Pi \cap S_{2}$ are tangent at $p$, the common tangent at $p$ has multiplicity 2. Since only this common tangent is a line in $\sigma$, the multiplicity of $\sigma$ in $\tau$ is at most 2 , and hence equal to 2 in case (i).

In case (ii) $(\ell \subset \Pi)$ we similarly suppose that $\ell$ is any line in the $y z$-plane not containing the origin $p$ and that $m$ is a line not lying in the $y z$-plane that meets $\ell$ at a point $q$. None of the four common tangents to $S_{1}$ and $S_{2}$ in the plane spanned by $\ell$ and $m$ is a line in $\sigma$, and there are four common tangents (counted with multiplicity) containing $q$. As before, there will be three such common tangents with the line $\overline{p q}$ having multiplicity 2 . This completes the proof in case (ii) as $\overline{p q}$ is in $\sigma$.

The proof in case (iii) is a consequence of Example 14 below.

Example 14. Suppose that the spheres $S_{1}$ and $S_{2}$ are tangent at the point $p=(0,0,0)^{T}$ with common tangent plane the $y z$-plane. Scaling the coordinates, we may assume that $S_{1}$ has centre $(1,0,0)^{T}$ with radius 1 and $S_{2}$ has centre $(-r, 0,0)^{T}$ with radius $|r|$. We forbid the values of $-1,0,1$ for $r$ : If $r=-1$, then the spheres coincide, $r=0$ is a degenerate sphere, and if $r=1$, then there is additional symmetry which we discuss in Section 3.2. We also assume that $\ell$ is the $z$-axis. Then the curve $\tau$ of lines tangent to $S_{1}$ and $S_{2}$ that also meet $\ell$ is cut out by the polynomials $p_{12}$, and

$$
\begin{gathered}
p_{03} p_{12}-p_{02} p_{13}+p_{01} p_{23}, \\
-p_{01}^{2}-2\left(p_{02} p_{12}+p_{03} p_{13}\right)+p_{12}^{2}+p_{13}^{2}+p_{23}^{2} \quad \text { and } \\
-r^{2} p_{01}^{2}+2 r\left(p_{02} p_{12}+p_{03} p_{13}\right)+p_{12}^{2}+p_{13}^{2}+p_{23}^{2} .
\end{gathered}
$$

Let $\sigma \subset \mathbb{G}$ be the set of lines in the $y z$-plane through the origin. As before, $\sigma$ is a component of the curve $\tau$ of common tangents to the spheres that meet the $z$-axis. The remaining components have degree at most 7 , when counted with their multiplicities. We invite the reader to check that under the following substitution:

$$
\begin{array}{ll}
p_{01}=2 \sqrt{r}\left(s^{2}+t^{2}\right)\left(s^{2}-t^{2}\right), & p_{12}=0, \\
p_{02}=4 \sqrt{r} s t\left(s^{2}+t^{2}\right), & p_{13}=2 r\left(s^{2}-t^{2}\right)^{2}, \\
p_{03}=(r-1)\left(s^{2}+t^{2}\right)^{2}, & p_{23}=4 r s t\left(s^{2}-t^{2}\right),
\end{array}
$$

the polynomials (2.1) vanish identically for all $[s, t] \in \mathbb{P}^{1}$. The polynomials in $s, t$ in (2.2) have no common factor, so this substitution defines a parametrized rational quartic curve $\rho$ in $\mathbb{G}$ which is a component of $\tau$ having degree 4 . The bound of 7 for the degrees of the components of $\tau-\sigma$ shows that $\rho$ has multiplicity 1 in $\tau$. Since the five quartic polynomials in $s, t$ of (2.2) are linearly independent, $\rho$ is a rational normal quartic curve in the hyperplane $p_{12}=0$ of Plücker space. 


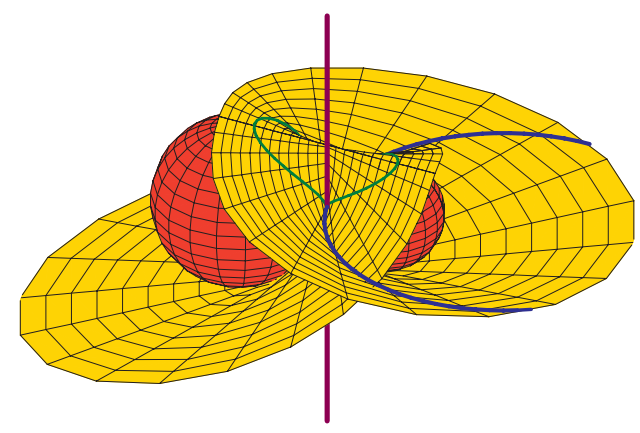

Fig. 4. An envelope.

We claim that $\tau=\sigma \cup \rho$, which implies that $\sigma$ has multiplicity 4 in $\tau$. To prove this, we first observe that the lines in $\rho$ are the column space of the matrix

$$
\left[\begin{array}{cccc}
s^{2}+t^{2} & 0 & 0 & -\sqrt{r}\left(s^{2}-t^{2}\right) \\
0 & 2 \sqrt{r}\left(s^{2}-t^{2}\right) & 4 \sqrt{r} s t & (r-1)\left(s^{2}+t^{2}\right)
\end{array}\right]^{T} .
$$

Note that no line of $\rho$ coincides with the $z$-axis.

Let $T$ be the envelope of $\tau$. Suppose that $\Pi$ is any plane containing the $z$-axis, other than the $y z$-plane which is the common tangent plane to $S_{1}$ and $S_{2}$. Then the intersection $T \cap \Pi$ consists of the $z$-axis and two remaining common tangents to the circles $\Pi \cap S_{1}$ and $\Pi \cap S_{2}$. We may suppose that $\Pi$ is defined by an equation $x=A y$. By (2.3) the lines in $\rho$ lying in $\Pi$ have parameter values satisfying $2 \sqrt{r}\left(s^{2}-t^{2}\right)=4 A \sqrt{r} s t$, so there are exactly two lines in $\rho$ that lie in $\Pi$. Furthermore, none of these lines coincides with the $z$-axis. Since any line in $\tau$ that does not lie in $\sigma$ will span such a plane $\Pi$ with the $z$-axis, we see that lines in $\tau$ not in $\sigma$ must lie in $\rho$, which completes the proof that $\sigma$ has multiplicity 4 in $\tau$.

In Fig. 4 we display the envelope $R$ of the rational normal curve $\rho$ when $r=2$. The envelope has been cut away to reveal both spheres. Drawn on the envelope are the two curves where the envelope is tangent to the spheres. The remaining arc of a circle is one component of the locus of self-intersection of the envelope, the other being the $z$-axis.

\subsection{The Degree of $\sigma$ Is 2}

Suppose now that the curve $\tau$ of common tangents to $S_{1}$ and $S_{2}$ that meet $\ell$ has a component $\sigma$ of degree 2. As in Example 7, there are three possibilities for the envelope $\Sigma$ of $\sigma$. We discuss possible configurations of the spheres and line for each; these possibilities correspond to cases (ii), (iii) and (iv) of Theorem 1.

(i) The envelope $\Sigma$ is a cone or cylinder. Because the lines on $\Sigma$ are tangent to the spheres $S_{1}$ and $S_{2}, \Sigma$ is circular and its axis $m$ contains the centres of the spheres. Since no line in $\mathbb{R}^{3}$ meets more than two lines on a circular cylinder, $\Sigma$ must 
be a cone with apex $p$ and $p \in \ell$. This gives case (ii) of Theorem 1: $S_{3}$ is any sphere inscribed in the cone $\Sigma$ whose centre (on $m$ ) is any point distinct from the apex or the centres of $S_{1}$ and $S_{2}$.

(ii) The envelope $\Sigma$ is the set of lines in a plane $\Pi$ tangent to a smooth conic $C$ in $\Pi$. Necessarily $\ell \subset \Pi$ and $\Pi \cap S_{1}=\Pi \cap S_{2}=C$, and $C$ is a circle. This gives case (iii) of Theorem 1: $S_{3}$ is any other sphere with $\Pi \cap S_{3}=C$.

(iii) The envelope $\Sigma$ is a smooth quadric surface $Q$ with $\sigma$ one of the rulings of $Q$. Then $\ell$ is a line in the other ruling. Since $Q$ contains the real line $\ell$, it is either a hyperbolic paraboloid or a hyperboloid of one sheet. As the lines in one ruling of $Q$ are tangent to the spheres $S_{1}$ and $S_{2}$, it cannot be a hyperbolic paraboloid. By Lemma 17 of [7], $Q$ is tangent to $S_{1}$ and $S_{2}$ and is a hyperboloid of revolution with axis $m$ containing the centres of $S_{1}$ and $S_{2}$. Then $\ell$ is tangent to both $S_{1}$ and $S_{2}$ and $Q$ is obtained by revolving $\ell$ around the line $m$ spanning the centres of $S_{1}$ and $S_{2}$. This gives case (iv) of Theorem 1: $S_{3}$ is any sphere inscribed in $Q$ whose centre (on $\mathrm{m}$ ) is any point different than the centres of $S_{1}$ and $S_{2}$.

The following proposition will be useful in Sections 2.6 and 3.

Proposition 15. A component of $\tau$ of degree 2 has multiplicity 1 in $\tau$. There is a unique component of degree 2 or of degree 1 with multiplicity 2 unless either the centres of $S_{1}$ and $S_{2}$ both lie on $\ell$ or $S_{1}$ and $S_{2}$ are symmetric with respect to $\ell$.

If the centres of $S_{1}$ and $S_{2}$ both lie on $\ell$, then $\tau$ has two components of degree 2, one of which degenerates into a component of degree 1 with multiplicity 2 if $S_{1}$ and $S_{2}$ are tangent to each other. If we choose coordinates so that $\ell$ is the $x$-axis, then $\tau$ also contains the one-dimensional linear subspaces

$$
p_{01}=p_{23}=p_{02} \mp i p_{03}=p_{12} \mp i p_{13}=0,
$$

each with multiplicity 2 . These parametrize lines in $\mathbb{P}^{3}$ that pass through a point of $\ell$ and one of the points at infinity $(0,0,1, \pm i)^{T}$ where the spheres are tangent to each other.

Two spheres in $\mathbb{P}^{3}$ meet in two conics. One is the imaginary spherical conic at infinity defined by $x_{0}=0$ and $x_{1}^{2}+x_{2}^{2}+x_{3}^{3}=0$, and the other lies in plane perpendicular to the line segment joining the centres of the spheres.

Proof. From the geometric description of the configuration of the spheres and of $\ell$ when there is a component of multiplicity 2 , there can only be one component of degree 2 or of degree 1 with multiplicity 2 in $\tau$, unless we are in one of two special cases: either (1) the centres of both spheres lie on $\ell$, or (2) $\ell$ lies in a plane (in $\mathbb{P}^{3}$ ) in which the spheres meet and $\ell$ also meets the apex of a cone tangent to both spheres. Since $\ell \subset \mathbb{R}^{3}$, this plane is perpendicular to the line segment joining the centres of the spheres and, moreover, the spheres are necessarily symmetric about $\ell$. In this case exactly one of the two components of degree 2 is real as either they meet in an imaginary conic and the cone tangent to the spheres with apex in the plane of symmetry is real, or vice versa. Also in this latter case, the remaining common tangents that meet $\ell$ form an irreducible quartic, illustrated in Fig. 7 and described in Theorem 18. 
Consider the first special case. Suppose that both centres lie on $\ell$, which we take to be the $x$-axis, and consider the intersection of $S_{1}$ and $S_{2}$ with a real plane $\Pi$ through $\ell$. These circles will have four common tangent lines consisting of two pairs symmetric with respect to $\ell$. Rotating these tangent lines about $\ell$ gives two cones or cylinders, one of which degenerates into a plane if $S_{1}$ and $S_{2}$ are tangent to each other. This gives two degree 2 components of $\tau$, or a degree 2 component and a degree 1 component with multiplicity 2 . Such spheres are the tangent to each other at the point $(0,0,1, \pm i)^{T}$ at infinity, with the tangent plane containing $\ell$ : this explains the two degree 1 components of $\tau$ having multiplicity 2 .

Now we show that a component $\sigma$ of degree 2 always has multiplicity 1. By Proposition 6, the lines lying in a plane $\Pi$ through $\ell$ or passing through a point $p \in \ell$ form a 2-plane in $\mathbb{G}$. In general, by Propositions 2 and 3 , there are four common tangent lines to $S_{1}$ and $S_{2}$ lying in $\Pi$ or passing through any point $p$ of $\ell$, so the corresponding 2-planes in $\mathbb{G}$ meet $\tau$ at four points counted with multiplicity.

Consider the three possibilities for a degree 2 component $\sigma$ of $\tau$ :

(i) When the envelope $\Sigma$ of $\sigma$ is a cone, two lines of $\sigma$ lie in any plane $\Pi$ through $\ell$, but there will be two other common tangents. This accounts for the four lines of $\tau$ lying in the 2-plane $\Pi$. Thus $\sigma$ must have multiplicity 1 , for otherwise both lines of $\sigma$ would have multiplicity 2 and there would be no others.

(ii) When the envelope consists of tangents to a conic $\Pi \cap S_{1}=\Pi \cap S_{2}$ in a plane $\Pi$ through $\ell$, two tangents in $\sigma$ meet a general point $p$ of $\ell$, but there will be two other common tangents to $S_{1}$ and $S_{2}$ meeting $p$. As before, this implies that $\sigma$ has multiplicity 1 .

(iii) Now suppose that $\ell$ is tangent to the spheres and $\sigma$ is one ruling of a hyperboloid of revolution. Every line $m$ of $\sigma$ meets $\ell$ and with it spans a plane $\Pi$. Unless $m \cap \ell$ is a point on one of the spheres, then $\Pi$ will contain four distinct common tangents to $S_{1}$ and $S_{2}$, thus two in addition to $m$ and $\ell$. Again, this implies that $\sigma$ has multiplicity 1 .

\subsection{The Degree of $\sigma$ Is 3}

We show that this possibility does not occur.

Proposition 16. The curve $\tau$ of common tangents to two spheres that meet a fixed line cannot have an irreducible component of degree 3.

Proof. Let $\sigma \subset \tau$ be a component of degree 3. Then either $\sigma$ is a plane cubic or else it spans a three-dimensional linear subspace of $\mathbb{P}^{5}$. Suppose that $\sigma$ is a plane cubic. As $\mathbb{G}$ is a quadric, it can only contain $\sigma$ if it contains the whole plane it spans. This plane corresponds either to all lines through a given point in $\mathbb{P}^{3}$ or to all lines contained in a given plane, but in both cases the lines tangent to a sphere in such a plane are parametrized by a conic, not a cubic, and so this case cannot occur.

Now suppose that $\sigma$ spans a three-dimensional subspace $H$ of $\mathbb{P}^{5}$. Note that $H \subset \Lambda_{\ell}$. There are two possibilities by Remark 4: either $H \cap \mathbb{G}$ is the set of lines meeting $\ell$ and 
another (uniquely defined) line $\ell^{\prime}$, or $H \cap \mathbb{G}$ is a cone with apex $p_{\ell}$ over a plane conic. In the first case, both $\ell$ and $\ell^{\prime}$ are real and $\sigma$ is a cubic curve of lines meeting $\ell$ and $\ell^{\prime}$ that are also tangent to two spheres. As shown in Section 5.1 of [7], this is impossible; the lines in a cubic in $\Lambda_{\ell} \cap \Lambda_{\ell^{\prime}}$ are tangent to at most one sphere. That determination was algebraic, and did not appeal to the real numbers.

Consider the last possibility, that $H \cap \mathbb{G}$ is a cone with apex $p_{\ell}$ over a plane conic. Since $\sigma$ is a cubic curve in $H \cap \mathbb{G}$, is must meet the apex and so $\ell$ is tangent to both spheres. Let $\Sigma$ be the envelope of $\sigma$, a cubic surface in $\mathbb{P}^{3}$. The curve $\gamma \subset S_{1}$ along which lines in $\sigma$ are tangent to $S_{1}$ is a component with multiplicity 2 of the (3,3)-curve $\Sigma \cap S_{1}$. This implies that $\gamma$ is a $(1,1)$-curve, and that $\Sigma \cap S_{1}$ contains components other than $\gamma$. These other components necessarily are the lines in $\sigma$ that lie in $S_{1}$. Such a line must contain the point where $\ell$ is tangent to $S_{1}$, and it is not real, so $\sigma$ contains its complex conjugate. As both meet $\ell$ and $\ell \not \subset S_{1}$, they meet $\ell$ at its point of tangency with $S_{1}$. Furthermore, these lines and $\ell$ lie in the tangent plane to $S_{1}$ at that point. This implies that $S_{2}$ is also tangent to $S_{1}$ at this same point. This configuration was studied in Example 14, where it was shown that the irreducible components of $\tau$ have degrees 1 and 4, and not 3 . This completes the proof of the impossibility of a cubic component.

\subsection{The Degree of $\sigma$ Is 4}

In Section 3 we show there are at most two spheres tangent to all the lines in a degree 4 curve of lines meeting a fixed line.

\subsection{The Degree of $\sigma$ Is 5}

This possibility also does not occur: if a component $\sigma$ of the curve $\tau$ of common tangents to the spheres $S_{1}$ and $S_{2}$ that also meet $\ell$ has degree 5, then the residual curve consisting of the other components of $\tau$ has degree 3. By Proposition 16, this degree 3 curve cannot be irreducible and reduced. Theorem 13 excludes all the possibilities that the degree 3 curve could split into lower degree components.

\subsection{The Degree of $\sigma$ Is 6}

We show that in most cases a union of components of $\tau$ having degree 6 determines the spheres $S_{1}$ and $S_{2}$.

Theorem 17. Let $\tau$ be the curve of common tangents to spheres $S_{1}$ and $S_{2}$ that also meet a fixed line $\ell$ and suppose that $\tau$ is reducible with a component $\rho$ either of degree 2 or of degree 1 with multiplicity 2 . Then the residual sextic $\sigma$ determines $S_{1}$ and $S_{2}$, except when their centres both lie on $\ell$.

By Proposition 15, if the centres of $S_{1}$ and $S_{2}$ lie on $\ell$, then $\tau$ has four components so that the residual sextic is reducible. 
Proof. Let $R, \Sigma$ and $T$ be, respectively, the envelopes of the curves $\rho, \sigma$ and $\tau$. Except when the centres of $S_{1}$ and $S_{2}$ lie on $\ell$ (which may be determined from $\Sigma$ ), we argue that we can determine the spheres $S_{1}$ and $S_{2}$ from $\sigma$.

In Section 2.2 we discussed three possibilities for a component of $\rho$ with degree 2 . The two cases (i) and (ii) of Theorem 13 for a component with degree 1 and multiplicity 2 are, respectively, degenerations of cases (i) and (ii) of Section 2.2. There will be five cases to consider for the geometry of $\rho$ and we treat each case separately.

In the first paragraph of each case we describe the number of lines in $\sigma$ meeting a general point of $\ell$ and the number of lines in $\sigma$ contained in a general plane $\Pi$ through $\ell$, together with some additional special geometry of that configuration. These descriptions will be different, showing that the sextic $\sigma$ determines each case. Recall from Propositions 2 and 3 that $\tau$ contains four lines through a general point $p$ of $\ell$ and four lines lying in a general plane $\Pi$ through $\ell$.

(i) Suppose that $R$ is a cone with apex $p$ lying on $\ell$, that $S_{1}$ and $S_{2}$ are inscribed in $R$ and that $\ell \notin \rho$, so that $\ell$ is tangent to neither $S_{1}$ nor $S_{2}$. (We treat the specialization $\ell \in \rho$ in (iv) below.) Then there will be a plane containing $\ell$ and the centres of $S_{1}$ and $S_{2}$ as well as two distinct planes through $\ell$ that are tangent to both $S_{1}$ and $S_{2}$. Since each line in $\rho$ meets $\ell$ at $p$ and two lie in any plane through $\ell$, there will be four lines in $\sigma$ meeting a general point of $\ell$ and two lines in $\sigma$ lying in a general plane $\Pi$ through $\ell$, counted with multiplicity. These observations remain valid if the configuration becomes degenerate so that $S_{1}$ and $S_{2}$ are tangent at $p$ and $\ell$ does not lie in their common tangent plane.

If, for every plane $\Pi$ through $\ell$, the lines $m_{\Pi}$ and $m_{\Pi}^{\prime}$ of $\sigma$ lying in $\Pi$ meet on $\ell$, then the centres of $S_{1}$ and $S_{2}$ both lie on $\ell$. (To see this, let $\Pi$ be a plane containing $\ell$ and the centres of $S_{1}$ and $S_{2}$.) In this case, $\sigma$ will have three components as detailed in Proposition 15: the two complex double lines (2.4) in $\mathbb{G}$, as well as the family of lines obtained by rotating $m_{\Pi}$ around $\ell$. Any sphere whose centre lies in $\ell$ and is tangent to one $m_{\Pi}$ will be tangent to all the lines in $\sigma$.

Now suppose that the centres do not lie on $\ell$. Then the two tangent planes to $S_{1}$ and $S_{2}$ through $\ell$ each contain a single line of $\sigma$ with multiplicity 2 (these are also double lines in $\rho$ ). These double tangent lines span a plane containing the centres of $S_{1}$ and $S_{2}$. There is a unique plane $\Pi$ through $\ell$ such that the two tangent lines contained in it intersect on $\ell$ - this plane is perpendicular to the plane containing $\ell$ and the centres of the two spheres. Thus we have two distinct planes containing the two centres and so their intersection gives the line $m$ passing through the two centres. Rotating the known double tangent lines around $m$ we obtain the cone, cylinder or double plane parametrized by the omitted degree 2 or degree 1 component of $\tau$, and thus $\tau$. By Theorem 9, this is sufficient to determine $S_{1}$ and $S_{2}$.

(ii) Suppose that $\rho$ consists of lines tangent to a smooth conic $C=\Pi \cap S_{1}=\Pi \cap S_{2}$ in a plane $\Pi$ through $\ell$, and that $\ell$ is not tangent to $C$. (We treat the specialization of $\ell$ tangent to $C$ in (v) below.) Any point $p$ of $\ell$ contains two lines from $\rho$ tangent to the conic, and so it contains two lines from $\sigma$. We also conclude that any plane through $\ell$ (other than $\Pi$ ) will have four lines from $\sigma$.

As in the proof of Theorem 9, this determines the spheres $S_{1}$ and $S_{2}$. Lastly, these observations remain valid if the positions of $S_{1}$ and $S_{2}$ become degenerate so that they are tangent to each other at the same point of the plane $\Pi$. 
(iii) Suppose now that we are in case (iii) of Section 2.2: $\ell$ is tangent to the spheres and $\rho$ is a ruling of the hyperboloid obtained by revolving $\ell$ about the line joining the centres of the spheres. Since $\ell \notin \rho$, we see that $\ell \in \sigma$. Then every point of $\ell$ contains a line in $\rho$ and every plane $\Pi$ through $\ell$ also contains a line in $\rho$. Thus any point $p$ of $\ell$ lies on three lines in $\sigma$ and any plane $\Pi$ through $\ell$ has three lines from $\sigma$. For both, the three include $\ell$ itself.

Suppose that a plane $\Pi$ through $\ell$ is tangent to one of the spheres. Then the lines of $\sigma$ in $\Pi$ consist of $\ell$ counted with multiplicity 2 and one other tangent line meeting $\ell$ at the point where it is tangent to that sphere. In this way we can determine the points at which the spheres are tangent to $\ell$. A general plane $\Pi$ through $\ell$ will contain two common tangent lines to $S_{1}$ and $S_{2}$ apart from $\ell$. From these lines and from the known points of the sphere's tangency with $\ell$, we can determine the circles $\Pi \cap S_{1}$ and $\Pi \cap S_{2}$. As before this is sufficient to determine $S_{1}$ and $S_{2}$.

(iv) The most intricate case is when the envelope $R$ of $\rho$ is a cone with apex $p$ tangent to both spheres with $\ell$ a ruling of $R$, so that $\ell \in \rho$. Thus $\ell$ is tangent to both spheres with common tangent plane $H$. We first observe that $\ell \in \sigma$. To see this, note that for any plane $\Pi$ through $\ell$ (except $H$ ), there will be two lines (besides $\ell$ ) in $\Pi \cap \Sigma$. Thus $H$ contains at least two lines from $\sigma$, counted with multiplicity. Since $H \cap \Sigma=\ell$ as $H$ is tangent to both spheres, we see that $\ell \in \sigma$. Thus for $\Pi$ through $\ell$ with $\Pi \neq H, \Pi \cap \Sigma$ has two lines, and $H$ is the unique plane such that $H \cap \Sigma$ is a single line. The plane $M$ through $\ell$ that is perpendicular to the tangent plane $H$ contains the centres of the spheres. As observed, $M \cap \Sigma$ contains two lines $m$ and $m^{\prime}$ besides $\ell$. The lines $\ell, m$ and $m^{\prime}$ are tangent to the equatorial circles $C_{1}:=M \cap S_{1}$ and $C_{2}:=M \cap S_{2}$ of the spheres. Since the fourth line tangent to the circles lies in the cone $R$ with apex $p$, the line through their centres (and $p$ ) meets the lines $m$ and $m^{\prime}$ at their intersection, and in fact bisects one of the angles there. Since there is another pair $C_{1}^{\prime}$ and $C_{2}^{\prime}$ of circles tangent to the three lines with the line connecting their centres bisecting the other angle, there are exactly two possibilities for the equatorial circles $C_{1}$ and $C_{2}$, and hence the spheres $S_{1}$ and $S_{2}$. This geometry in the plane $M$ is indicated in the picture on the left of Fig. 5 .

The envelope $\sigma$ selects the correct pair. Indeed, let $S_{1}, S_{2}$ and $S_{1}^{\prime}, S_{2}^{\prime}$ be the two possible pairs of spheres, and consider another plane $\Pi$ through $\ell$, but distinct from both $M$ and the tangent plane $H$. It will intersect these four spheres in four circles, each tangent to $\ell$. However, only two can be tangent to the other two

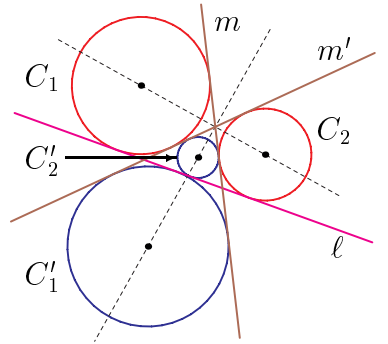

The plane $M$

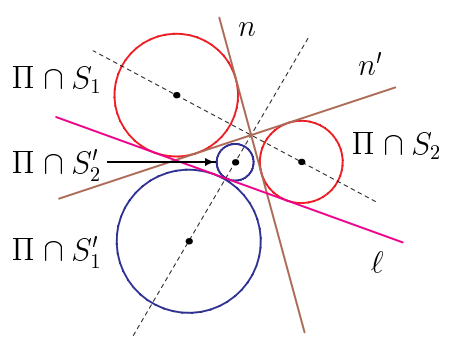

The plane $\Pi$

Fig. 5. Configurations within planes. 


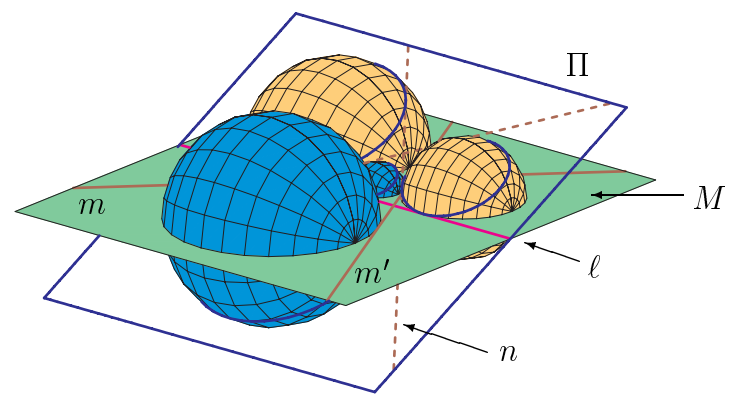

Fig. 6. Configuration of the spheres.

lines $n, n^{\prime}$ in $\sigma$ lying in $\Pi$. In this way we see that $\sigma$ determines $S_{1}$ and $S_{2}$. The configuration within $\Pi$ is indicated in the picture on the right in Fig. 5, while the configurations of the lines in $M$, the two pairs of spheres and their intersections with $\Pi$ (drawn in outline) is shown in Fig. 6 . Note that the line $n$ is not tangent to the sphere $S_{1}^{\prime}$ (the sphere in the foreground).

(v) In the last case, $\rho$ consists of lines tangent to a smooth conic $C=\Pi \cap S_{1}=\Pi \cap S_{2}$ in a plane $\Pi$ through $\ell$, and $\ell$ is tangent to $C$ at a point $q$. We first observe that $\ell \in \sigma$. To see this, note that for any point $p \neq q$ of $\ell$ there will be two lines in $\sigma$ through $p$ (besides $\ell$ ). Thus $\sigma$ will contain at least two lines that meet $q$, counted with multiplicity. Since $\ell$ is the only line tangent to both spheres through the point $q$, we conclude that $\ell \in \sigma$. Thus $\sigma$ has three lines meeting a point $p \neq q$ of $\ell$ and $q$ is the unique point of $\ell$ that meets only one line from $\sigma$.

We complete the proof in this case by noting that every plane $H$ through $\ell$ (other than $\Pi$ ) will have three lines from $\sigma$. This determines the $H \cap S_{1}$ and $H \cap S_{2}$ as they are both tangent to $\ell$ at the point $q$. We conclude once again that $\sigma$ determines the spheres $S_{1}$ and $S_{2}$, and this completes the proof.

\subsection{The Degree of $\sigma$ Is 7}

As shown in Section 2.1, any degree 1 component of $\tau$ has multiplicity at least 2. Thus there cannot be a component of degree 7 .

\section{Quartics}

We describe completely the configurations of the spheres $S_{1}$ and $S_{2}$ in the case when the curve $\tau$ has an irreducible quartic component $\sigma$. We also show that there can be no other spheres tangent to all the lines in $\sigma$. This uses both algebraic (in complex $\mathbb{P}^{3}$ ) and geometric (in $\mathbb{R}^{3}$ ) arguments.

Theorem 18. Let $\sigma \subset \mathbb{G}$ be an irreducible real quartic curve parametrizing lines meeting a fixed line $\ell$ and tangent to a fixed sphere $S_{1}$. If there is another sphere $S_{2}$ 


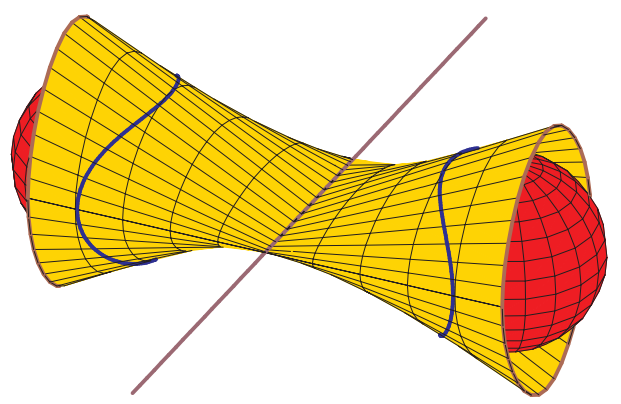

Fig. 7. Symmetric spheres.

tangent to all these lines, then either $S_{1}$ and $S_{2}$ are tangent to each other and to $\ell$ at the same point or $S_{1}$ and $S_{2}$ are symmetric with respect to $\ell$ or both. In particular, there cannot be a third sphere tangent to all the lines in $\sigma$.

The first case of this theorem is the situation of Example 14, and the second is illustrated in Fig. 7.

For the last statement in Theorem 18, note that if three spheres are tangent to each other at a point $p$, then one sphere lies inside the other and the set of common tangents are the lines tangent to the spheres at the point $p$, which has degree 1 and not 4 . Also we cannot have three distinct spheres that are pairwise symmetric around $\ell$.

Our proof considers the possibilities for such a degree 4 component of $\tau$, ruling out most of them. To begin, there are three possibilities for the dimension of the linear span $H$ of the curve $\sigma$ in Plücker space, and we treat each separately below. Note that $H \subset \Lambda_{\ell}$.

\subsection{The Dimension of $H$ Is 2}

In this case, $\sigma$ is a plane quartic. Since $\mathbb{G}$ is a quadric, it must contain the plane $H$, but the lines lying in such a plane that are tangent to a sphere are parametrized by a conic and not a quartic. We conclude that this case is impossible.

\subsection{The Dimension of $H$ Is 3}

If $\sigma$ spans a three-dimensional subspace $H$, then by Remark 4 there are two possibilities: either $H \cap \mathbb{G}$ is the set of lines meeting $\ell$ and another (uniquely defined) line $\ell^{\prime}$, or $H$ is tangent to $\mathbb{G}$ at the point $p_{\ell}$. In the first case, both $\ell$ and $\ell^{\prime}$ are real and $\sigma$ is a quartic curve of lines meeting them that are also tangent to two spheres. This situation was studied in [7]: by Theorem 16 of [7] $\ell^{\prime}$ cannot lie in affine space because those cases do not give a quartic curve of common tangents and transversals, and by Theorem 20 of [7], $\ell^{\prime}$ must be the line at infinity perpendicular to $\ell$ and the two spheres must be symmetric with respect to $\ell$. This is the second case of Theorem 18. Furthermore, Theorem 20 of [7] also implies that there is no other sphere tangent to every line in $\sigma$. 
Suppose now that $H$ is tangent is to $\mathbb{G}$ at $p_{\ell}$. Let $\Lambda$ be another hyperplane through $H$ such that $H=\Lambda_{\ell} \cap \Lambda$. Then the curve $\sigma$ is the intersection of $\Lambda_{\ell}, \Lambda, \mathbb{G}$ and either the quadratic surface given by $\wedge^{2} S_{1}$ or the surface given by $\wedge^{2} S_{2}$. In particular, if $h$ is a linear form defining $H$ in $\Lambda_{\ell}$, then there is a linear form $k$ such that the quadratic form given by $\wedge^{2} S_{2}$ is a linear combination of the quadratic form given by $\wedge^{2} S_{1}$, the Plücker relation (1.1) defining $\mathbb{G}$ and $h k$, modulo $\Lambda_{\ell}$.

Suppose that $\ell$ is the $x$-axis so that $\Lambda_{\ell}$ is the hyperplane $p_{23}=0$. For each $i=1,2$, let $\left(x_{i}, y_{i}, z_{i}\right)^{T}$ be the centre of $S_{i}$ and let $r_{i}$ be its radius. Let $q_{1}$ and $q_{2}$ be the quadratic forms given by $\wedge^{2} S_{1}$ and $\wedge^{2} S_{2}$ with $p_{23}$ set equal to zero and let $g$ be the Plücker relation with $p_{23}=0$. These define the restrictions of the quadratic forms $\wedge^{2} S_{1}, \wedge^{2} S_{2}$ and the Plücker relation to $\Lambda_{\ell}$. Let $h$ be the equation of $H$ in $\Lambda_{\ell}$. By the remark at the end of the last paragraph, there exists a real linear form $k$ on $\Lambda_{\ell}$ and real numbers $\lambda, \mu$ and $v$ such that $\lambda q_{1}+\mu q_{2}+v g=h k$. Furthermore, as either of $q_{1}$ or $q_{2}$ may be written in terms of the other forms, we have $\lambda, \mu \neq 0$.

Thus the quadratic form $\lambda q_{1}+\mu q_{2}+v g$ has rank 2 and therefore every $3 \times 3$ minor of its representation matrix $M$ vanishes. We argue that this is impossible. Here is $M$ :

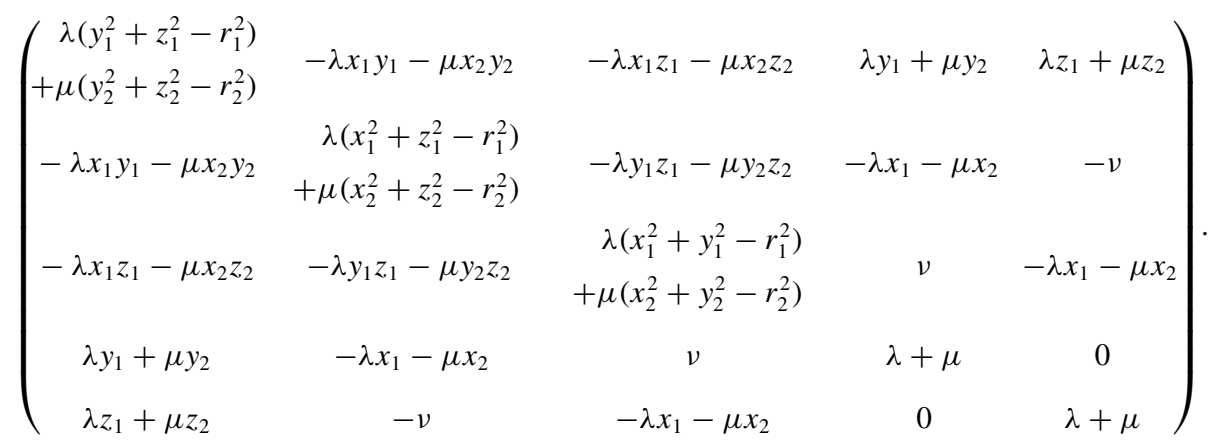

Since $H$ contains the point $p_{\ell}=(1,0,0,0,0,0)^{T}$, the linear form $h$ has no $p_{01}$ term, therefore $h k$ has no $p_{01}^{2}$ term. Thus the upper left entry of $M$ vanishes, giving the equation

$$
\lambda\left(y_{1}^{2}+z_{1}^{2}-r_{1}^{2}\right)+\mu\left(y_{2}^{2}+z_{2}^{2}-r_{2}^{2}\right)=0 .
$$

Let $M_{a b c, d e f}$ be the minor formed by the rows $a b c$ and the columns def. Since $M_{145,145}=$ $(\lambda+\mu)\left(\left(\lambda y_{1}+\mu y_{2}\right)^{2}+\left(\lambda z_{1}+\mu z_{2}\right)^{2}\right)=0$, we have two cases to consider.

Case 1: Suppose that $\lambda+\mu=0$. Scaling, we may assume that $\lambda=1$ and $\mu=-1$. Then $M_{234,345}=v\left(v^{2}+\left(x_{1}-x_{2}\right)^{2}\right)$ and $M_{235,345}=\left(x_{2}-x_{1}\right)\left(v^{2}+\left(x_{1}-x_{2}\right)^{2}\right)$. Since $x_{1}, x_{2}$ and $v$ are real, this implies that $v=0$ and $x_{1}=x_{2}$. Setting $x:=x_{1}=x_{2}$, the matrix $M$ becomes

$$
\left(\begin{array}{ccccc}
0 & -x y_{1}+x y_{2} & -x z_{1}+x z_{2} & y_{1}-y_{2} & z_{1}-z_{2} \\
-x y_{1}+x y_{2} & z_{1}^{2}-z_{2}^{2}+r_{2}^{2}-r_{1}^{2} & -y_{1} z_{1}+y_{2} z_{2} & 0 & 0 \\
-x z_{1}+x z_{2} & -y_{1} z_{1}+y_{2} z_{2} & y_{1}^{2}-y_{2}^{2}+r_{2}^{2}-r_{1}^{2} & 0 & 0 \\
y_{1}-y_{2} & 0 & 0 & 0 & 0 \\
z_{1}-z_{2} & 0 & 0 & 0 & 0
\end{array}\right) .
$$


Since the spheres are not concentric, at least one of $y_{1} \neq y_{2}$ or $z_{1} \neq z_{2}$ holds. Suppose that $y_{1} \neq y_{2}$. Then the equations $0=M_{124,124}=M_{134,134}=M_{124,134}$ imply that the entries not in the first row or column vanish, that is

$$
z_{1}^{2}-z_{2}^{2}+r_{2}^{2}-r_{1}^{2}=y_{1}^{2}-y_{2}^{2}+r_{2}^{2}-r_{1}^{2}=y_{1} z_{1}-y_{2} z_{2}=0 .
$$

Subtracting the first from (3.1) gives $0=y_{1}^{2}-y_{2}^{2}$ and therefore $y_{1}=-y_{2}$. With the third, we conclude that $z_{1}=-z_{2}$ and thus $r_{1}=r_{2}$. (We reach the same conclusion from $z_{1} \neq z_{2}$.)

Thus the spheres are symmetric with respect to the $x$-axis, the configuration of Fig. 7. That envelope corresponds to a curve of degree 4 whose linear span has the form $\Lambda_{\ell} \cap \Lambda_{\ell^{\prime}}$, and so it cannot be the curve $\sigma$. The other components are two conics as described in the proof of Proposition 15: one is the ruling of the cone over the spheres with the apex the point of symmetry, and the other consists of lines in the $x z$-plane tangent to the conic along which the spheres meet, and if the spheres are tangent, the components degenerate to a line of multiplicity 4. (This is the case $r=1$ in Example 14.) This gives a contradiction to the existence of $\sigma$ when $\lambda+\mu=0$.

Case 2: Suppose that $\lambda+\mu \neq 0$. Then $\lambda y_{1}+\mu y_{2}=\lambda z_{1}+\mu z_{2}=0$, since these are real numbers and the sum of their squares is zero. Since $M_{145,245}=-(\lambda+\mu)^{2}\left(\lambda x_{1} y_{1}+\right.$ $\left.\mu x_{2} y_{2}\right), M_{145,345}=-(\lambda+\mu)^{2}\left(\lambda x_{1} z_{1}+\mu x_{2} z_{2}\right)$ and $M_{245,345}=-(\lambda+\mu)^{2}\left(\lambda y_{1} z_{1}+\mu y_{2} z_{2}\right)$, we obtain

$$
\lambda x_{1} y_{1}+\mu x_{2} y_{2}=\lambda x_{1} z_{1}+\mu x_{2} z_{2}=\lambda y_{1} z_{1}+\mu y_{2} z_{2}=0 .
$$

Besides scaling $\lambda$ and $\mu$ by a common scalar, the only solutions to these five equations are

$$
\begin{array}{cccc}
x_{1}=x_{2}, & y_{1}=y_{2}=0, \quad z_{1}=-\mu, & z_{2}=\lambda, \\
x_{1}=x_{2}, & z_{1}=z_{2}=0, \quad y_{1}=-\mu, & y_{2}=\lambda, & \text { or } \\
y_{1}=y_{2}=z_{1}=z_{2}=0 . &
\end{array}
$$

Either of the first two solutions give $M_{245,245}-M_{345,345}= \pm \lambda \mu(\lambda+\mu)^{3}$, which leads to one of the excluded cases $\lambda=0, \mu=0$ or $\lambda+\mu=0$.

The third solution implies that the centres of $S_{1}$ and $S_{2}$ lie on the $x$-axis. As in Proposition 15 and in Theorem 17, $\tau$ will only have components of degrees 1 and 2 . This concludes the proof of the impossibility of a degree 4 component spanning a 3 -plane in Plücker space that is tangent to the Grassmannian.

\subsection{The Dimension of $H$ Is 4}

The remaining possibility is that $\sigma$ spans a four-dimensional subspace. As $\sigma$ has degree 4 , it is necessarily a rational normal quartic curve. There are two possibilities for the geometry of the curve $\gamma \subset S_{1}$ along which the lines of $\sigma$ are tangent to $S_{1}$. These lead to restrictions on the possible configurations of $S_{1}$ and $\ell$. We then consider planes $\Pi$ through $\ell$ that are tangent to $S_{1}$ and contain lines from $\sigma$ (these are determined from $\sigma$ ). From this analysis, we see that the only possibility for there to be a rational quartic $\sigma$ of lines tangent to two spheres that also meet a fixed line $\ell$ is when the spheres are tangent 
to each other and to $\ell$ at the same point, and thus $\sigma$ is the degree 4 component studied in Example 14.

First, let $\Sigma \subset \mathbb{P}^{3}$ be the envelope of the rational curve $\sigma$, a rational ruled surface of degree 4. The lines in $\sigma$ which are not contained in $S_{1}$ are tangent to $S_{1}$ at well-defined points, and the closure of these points of tangency forms a curve $\gamma$ lying on $S_{1}$. Since $\gamma$ is a real component of multiplicity 2 in the $(4,4)$-curve $\Sigma \cap S_{1}$, either $\gamma$ has bidegree $(1,1)$, so it is a plane conic, or it has bidegree $(2,2)$. When it has bidegree $(2,2)$, we must have that $\Sigma \cap S_{1}=\gamma$, in particular, $\sigma$ contains no lines lying in $S_{1}$. When $\gamma$ has bidegree $(1,1), \Sigma \cap S_{1}$ properly contains $\gamma$, and the residual curve has bidegree $(2,2)$. Thus it is the union of lines from the rulings of $S_{1}$, and so $\sigma$ contains a complex conjugate pair from each ruling of $S_{1}$. The association $\gamma \ni m \cap S_{1} \mapsto m \in \sigma$ is the restriction of the birational map $\Phi: S_{1} \rightarrow \mathbb{G}$ studied in Section 1.4 to $\gamma$, so that $\gamma$ is also a rational curve. Irreducible rational curves of bidegree $(2,2)$ on a quadric have a single singularity that is necessarily a simple node or a cusp. (See the discussion in Section 2 of [7] and the references therein.)

Lemma 19. There are two possibilities for the configuration of $S_{1}, \ell$ and $\gamma$.

(i) The curve $\gamma$ has bidegree $(1,1)$ and does not contain any point where $\ell$ meets $S_{1}$ or where a plane through $\ell$ is tangent to $S_{1}$.

(ii) The line $\ell$ is tangent to the sphere $S_{1}$, the curve $\gamma$ has bidegree $(2,2)$ and is singular at this point of tangency.

Proof. A point where $\ell$ meets $S_{1}$ or where a plane through $\ell$ is tangent to $S_{1}$ is a special point. Either $\gamma$ has bidegree $(1,1)$ or bidegree $(2,2)$, and either $\ell$ is tangent to $S_{1}$ or it is not. Suppose $\gamma$ has bidegree $(1,1)$ so that it has degree 2 . By the degree calculation of Lemma $8, \gamma$ cannot contain any of the special points, proving case (i).

Suppose that $\gamma$ has bidegree $(2,2)$, so that it has degree 4. By Lemma $8, \gamma$ must contain a special point. In particular, if $\ell$ is tangent to $S_{1}$ at the point $p$, then $p$ has multiplicity 2 in $\gamma$-that is, $p$ is the singular point of $\gamma$.

We exclude the remaining case of $\gamma$ having bidegree $(2,2)$ and $\ell$ not tangent to $S_{1}$. By Lemma 8(i), either $\gamma$ contains all four special points and is nonsingular at each, or $\gamma$ contains three of the four special points and is singular at one of the three. If $\gamma$ is singular at a special point $q$, then mult $q \geq 2$. Let $\Pi$ be the tangent plane to $S_{1}$ at $q$. Then the intersection multiplicity of $\Pi$ with $\gamma$ at this point is at least

$$
2 \cdot \operatorname{mult}_{q} \gamma \geq 4
$$

The factor 2 is due to the tangency of $\Pi$ at $\gamma$. Since $\gamma$ has degree 4 , its total intersection multiplicity with $\Pi$ is 4 . This implies that $\Pi \cap \gamma=q$. Since the tangent plane at any special point contains two others, $\gamma$ cannot be singular at a special point.

Suppose now that $\gamma$ contains four special points and is thus non-singular at each. Then the singular point $q$ of $\gamma$ is not a special point, and so the rational map $\Phi$ of (1.6) is regular at $q$ (well defined on a neighbourhood of $q$ ). It follows that the image of the singular point $q$ of $\gamma$ under $\Phi$ is a singular point of the image $\sigma$ of $\gamma$. Since any rational normal curve, and in particular $\sigma$, is smooth, this contradiction excludes this case. 


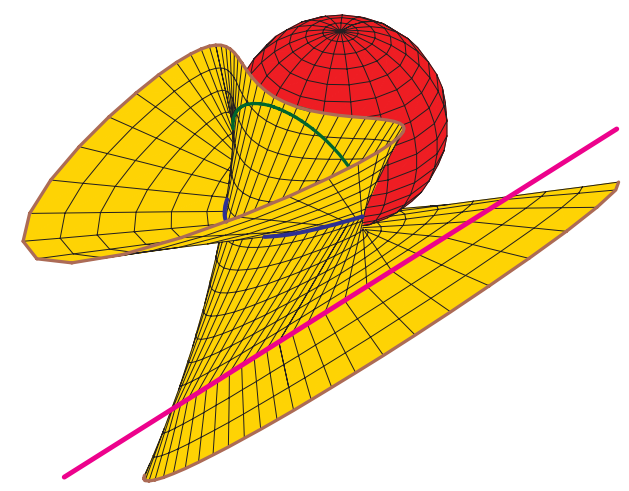

Fig. 8. Envelope of a quartic component.

Figure 8 displays the envelope of a degree 4 component whose curve $\gamma$ of tangency satisfies (i) in Lemma 19. An envelope corresponding to (ii) is illustrated in Fig. 4.

We complete the proof of Theorem 18 by considering special lines in $\sigma$ lying in planes $\Pi$ through $\ell$. This allows us to distinguish the two cases for the curve $\gamma$ given in Lemma 19, and then to show that the only possibility for two spheres to be tangent to the lines of $\sigma$ is for the spheres to be tangent to each other and to $\ell$ at the same point, the configuration of Example 14.

We first review some complex Euclidean analytic geometry. Suppose that $\ell$ is the $x$-axis. For $\lambda, \mu \in \mathbb{C}$ satisfying $\lambda^{2}+\mu^{2}=1$, consider the plane through $\ell$ given by the equation $\lambda y=\mu z$. All planes through $\ell$ have this form, except for the two planes $y= \pm i z$, and these two are independent of coordinate choice. Euclidean coordinates for the plane $\Pi: \lambda y=\mu z$ are given by $x$ and $v:=\mu y+\lambda z$. Suppose that $S_{1}$ is a sphere with centre $\left(x_{0}, y_{0}, z_{0}\right)$ and radius $r$. Then $\Pi \cap S_{1}$ is the circle

$$
\left(x-x_{0}\right)^{2}+\left(v-v_{0}\right)^{2}=r^{2}-y_{0}^{2}-z_{0}^{2}+v_{0}^{2},
$$

where $v_{0}=\mu y_{0}+\lambda z_{0}$. The plane $\Pi$ is tangent to $S_{1}$ when $v_{0}^{2}=y_{0}^{2}+z_{0}^{2}-r^{2}$, with the circle degenerating to the two lines $\left(x-x_{0}\right)= \pm i\left(v-v_{0}\right)$ with slopes $\pm i$. We remark that a line in such a plane with slope $\pm i$ is tangent to a circle only when the circle is degenerate.

Consider the planes $\Pi$ through $\ell$ having an equation of the form $\lambda y=\mu z$ with $\lambda^{2}+\mu^{2}=1$ that contain a line from $\sigma$ with slope $i$ or $-i$. Call such a plane distinguished. Note that the distinguished planes are determined by $\sigma$. Since distinguished planes are tangent to the sphere $S_{1}$, there are at most two. We consider the three cases of two, one or zero distinguished planes through $\ell$. Recall that if $\gamma$ has bidegree $(2,2)$, then $\Sigma \cap S_{1}$ necessarily equals $\gamma$ and there are no lines from $\sigma$ lying in $S_{1}$. Thus there can be no distinguished planes if $\gamma$ has bidegree $(2,2)$.

Two Distinguished Planes. We must be in case (i) of Lemma 19. Furthermore, the line $\ell$ does not contain the centre of $S_{1}$, for then no plane through $\ell$ of the form $\lambda y=\mu z$ with $\lambda^{2}+\mu^{2}=1$ is tangent to $S_{1}$ (tangent planes to $S_{1}$ through a line containing its centre have the form $y= \pm i z$ ). Conversely, if we are in case (i) of Lemma 19 and $\ell$ is neither 
tangent to $S_{1}$ nor contains the centre of $S_{1}$, then any line in $\sigma$ lying in a tangent plane $\Pi$ to $S_{1}$ through $\ell$ must have slope $\pm i$ : otherwise such a line in $\sigma$ meets $S_{1}$ only at the point $p$ of tangency of $\Pi$ to $S_{1}$, and thus $p \in \gamma$, which contradicts (i) of Lemma 19.

Consider lines in $\sigma$ lying in a distinguished plane $\Pi$. If there is only a single such line $m$, then its complex conjugate line $\bar{m}$ also lies in $S_{1}$ and is also in $\sigma$. Necessarily $\bar{m} \subset \bar{\Pi}$, the plane complex conjugate to $\Pi$. Since $m$ is the only such line in $\Pi$, we have that $\Pi \neq \bar{\Pi}$. As $m$ and $\bar{m}$ are complex conjugate, they lie in different rulings of $S_{1}$, and therefore meet in a point $p$ (necessarily real) of $S_{1}$. Note that $p$ lies in the intersection of $\Pi$ with $\bar{\Pi}$, which is $\ell$. Furthermore, the plane $H$ spanned by $m$ and $\bar{m}$ is tangent to $S_{1}$ at $p$.

If we have a second sphere tangent to all the lines of $\sigma$, then it is also tangent to $H$ at $p$. However, this configuration of the spheres and line $\ell$ is exactly that of Theorem 13(i) (see also (ia) in Fig. 1). In this case the lines in $H$ through $p$ form a component of degree 1 and multiplicity 2 in the curve $\tau$ of tangents to the two spheres that meet $\ell$. By Proposition 15, the residual sextic is irreducible as $\ell$ neither contains the centres of the spheres nor are they symmetric about $\ell$. However, this contradicts the existence of the quartic component $\sigma$. Observe that this argument only depends upon there being exactly one line from $\sigma$ in one of two tangent planes to $S_{1}$ through $\ell$.

Now suppose that $\sigma$ contains two lines in each distinguished plane $\Pi$. Since the two lines from $\sigma$ lying in a distinguished plane meet at the point of tangency, $\sigma$ determines two planes through $\ell$ tangent to $S_{1}$ and the points of tangency. These data determine the sphere $S_{1}$ and there is no possibility for a second sphere.

One Distinguished Plane. Now suppose that there is a unique distinguished plane $\Pi$. Then $\gamma$ has bidegree $(1,1)$ and $\ell$ does not contain the centre of $S_{1}$. Since $\sigma$ must contain lines from each ruling of $S_{1}$ and these must lie in a plane tangent to $S_{1}$ through $\ell$, we conclude that $\sigma$ contains both lines in $\Pi$ lying in $S_{1}$. Furthermore, $\Pi$ is real as otherwise $\bar{\Pi}$ is a different distinguished plane. Since the two lines from $\sigma$ meet at the point $p$ of tangency of $S_{1}$ to $\Pi$, this point and $\Pi$ are determined by $\sigma$.

If there is a second sphere tangent to all the lines from $\sigma$, it is necessarily tangent to $\Pi$ and hence also to $S_{1}$ at the point $p$. If $\ell$ is not tangent to $S_{1}$, this is the configuration of Theorem 13(ii) (see also (ib) in Fig. 1), and, as the spheres are not symmetric about $\ell$, Proposition 15 leads to a contradiction as before. On the other hand, $\ell$ cannot be tangent to $S_{1}$, for then this is the configuration of Example 14, and in that case lines lying in the common tangent plane had real slopes whereas the lines here have imaginary slopes.

No Distinguished Planes. Suppose that no plane through $\ell$ of the form $\lambda y=\mu z$ with $\lambda^{2}+\mu^{2}=1$ contains a line from $\sigma$ with slope $i$ or $-i$.

If the curve $\gamma$ has bidegree $(1,1)$, then $\ell$ necessarily contains the centre of $S_{1}$, as $\sigma$ contains lines lying in $S_{1}$ and these must lie in a plane through $\ell$ that is tangent to $S_{1}$. Otherwise $\ell$ is tangent to $S_{1}$ at the singular point of the $(2,2)$-curve $\gamma$. We distinguish these two cases by considering a plane $\Pi: y= \pm i z$ through $\ell$, which is independent of the choice of coordinates (up to complex conjugation). If the centre of $S_{1}$ lies on $\ell$, then $\Pi \cap S_{1}$ will be two lines meeting at the point $(0,0,1, \pm i)^{T}$ at infinity. Since $\gamma$ does not contain the point of tangency, at least one of these lines lies in $\sigma$. As argued in the case 
of two distinguished planes, we cannot have $\sigma$ containing only one of these lines, so $\gamma$ must contain both lines, and they meet at this point. On the other hand, if $\ell$ is tangent to $S_{1}$, then $\Pi \cap S_{1}$ will be a smooth conic containing the point $(0,0,1, \pm i)^{T}$ at infinity, and so the tangents in $\sigma$ lying in $\Pi$ cannot meet at this point. Thus we may distinguish the two cases when there are no distinguished planes.

Suppose that $\gamma$ has bidegree $(1,1)$ so that the lines from $\sigma$ in the plane $y= \pm i z$ contain the point $(0,0,1, \pm i)^{T}$. Then the line $\ell$ necessarily contains the centre of $S_{1}$. Thus if there are two spheres tangent to all the lines from $\sigma$, then their centres must lie on $\ell$. By Proposition 15, the curve $\tau$ of tangents to the two spheres that also meet $\ell$ will then have four components, none of which has degree more than 2 , so this case does not occur.

We finally conclude that $\ell$ is tangent to the sphere $S_{1}$ and $\gamma$ has bidegree $(2,2)$. Since this determination depends only upon $\sigma$, we conclude that the other sphere $S_{2}$ is also tangent to $\ell$. The points of tangency can be different or coincide, and the planes of tangency can be different or coincide. If the spheres are tangent to $\ell$ at the same point with the same tangent plane, then we have the configuration of Example 14. Thus the curve $\tau$ of common tangents to the spheres that meet $\ell$ consists of $\sigma$ together with the lines in the common tangent plane through the point of tangency, a component of multiplicity 4. By Theorem 9, this determines $S_{1}$ and $S_{2}$; in particular, there cannot be a third sphere.

We complete the proof of Theorem 18 and thus of Theorem 1 by disposing of the three remaining possibilities. If the spheres are tangent to $\ell$ at different points, but with the same tangent plane, then we have the configuration of (iv) in the proof of Theorem 17. If the points of tangency coincide, but the tangent planes are distinct, this is the configuration of (v) in the proof of Theorem 17. If the points of tangency and the tangent planes are all distinct, then this is the configuration of Section 2.2(iii). In each of these cases the curve $\tau$ of common tangents to the spheres that meet $\ell$ has a component of degree 2 , and, by Proposition 15, the rest of $\tau$ consists of an irreducible sextic, contradicting our assumption of a quartic component $\sigma$.

\section{References}

1. P.K. Agarwal, B. Aronov, and M. Sharir, Computing envelopes in four dimensions with applications, SIAM J. Comput. 26 (1997), 1714-1732.

2. C. Borcea, X. Goaoc, S. Lazard, and S. Petitjean, Common tangents to spheres in $\mathbb{R}^{3}, 2004$.

3. D. Cox, J. Little, and D. O'Shea, Ideals, Varieties, Algorithms: An Introduction to Computational Algebraic Geometry and Commutative Algebra, UTM, Springer-Verlag, New York, 1992.

4. P. Griffiths and J. Harris, Principles of Algebraic Geometry, Wiley, New York, 1978.

5. I.G. Macdonald, J. Pach, and T. Theobald, Common tangents to four unit balls in $\mathbb{R}^{3}$, Discrete Comput. Geom. 26(1) (2001), 1-17.

6. G. Megyesi, Lines tangent to four unit spheres with coplanar centres, Discrete Comput. Geom. 26(4) (2001), 493-497.

7. G. Megyesi, F. Sottile, and T. Theobald, Common transversal and tangents to two lines and two spheres in $\mathbb{P}^{n}$, Discrete Comput. Geom. 30 (2003), 543-571.

8. M. Pellegrini, Ray shooting and lines in spaces, in The CRC Handbook of Discrete and Computational Geometry (J.E. Goodman and J. O’Rourke, eds.), CRC Press, Boca Raton, FL, 1997, pp. 599-614.

9. H. Pottmann and J. Wallner, Computational Line Geometry, Springer-Verlag, Berlin, 2001. 
10. F. Sottile, From enumerative geometry to solving systems of equations, in Computations in Algebraic Geometry with Macaulay 2 (D. Eisenbud, D. Grayson, M. Stillman, and B. Sturmfels, eds.), ACM, No. 8, Springer-Verlag, New York, 2001, pp. 101-129.

11. F. Sottile and T. Theobald, Real lines tangent to $2 n-2$ quadrics in $\mathbb{R}^{n}$, Proc. $A M S$, to appear.

12. T. Theobald, An enumerative geometric framework for algorithmic line problems in $\mathbb{R}^{3}$, SIAM J. Comput. 31 (2002), 1212-1228.

13. T. Theobald, Visibility computations: from discrete algorithms to real algebraic geometry, in Proc. DIMACS Workshop on Algorithmic and Quantitative Aspects of Real Algebraic Geometry in Mathematics and Computer Science, 2002, to appear.

Received April 22, 2003, and in revised form September 7, 2004. Online publication March 30, 2005. 